\title{
Reduced fluid models for self-propelled particles interacting through alignment
}

\author{
Mihai Bostan \\ Institut de Mathématiques de Marseille, \\ UMR 7373, CNRS, Centrale Marseille, \\ Aix Marseille Université, \\ Château Gombert 39 rue F. Joliot Curie, \\ 13453 Marseille Cedex 13, France \\ mihai.bostan@univ-amu.fr \\ Jose Antonio Carrillo* \\ Department of Mathematics, Imperial College London, \\ London SW7 2AZ, UK \\ carrillo@imperial.ac.uk
}

Received 13 January 2017

Revised 26 February 2017

Accepted 28 February 2017

Published 18 May 2017

Communicated by N. Bellomo and F. Brezzi

\begin{abstract}
The asymptotic analysis of kinetic models describing the behavior of particles interacting through alignment is performed. We will analyze the asymptotic regime corresponding to large alignment frequency where the alignment effects are dominated by the selfpropulsion and friction forces. The former hypothesis leads to a macroscopic fluid model due to the fast averaging in velocity, while the second one imposes a fixed speed in the limit, and thus a reduction of the dynamics to a sphere in the velocity space. The analysis relies on averaging techniques successfully used in the magnetic confinement of charged particles. The limiting particle distribution is supported on a sphere, and therefore we are forced to work with measures in velocity. As for the Euler-type equations, the fluid model comes by integrating the kinetic equation against the collision invariants and its generalizations in the velocity space. The main difficulty is their identification for the averaged alignment kernel in our functional setting of measures in velocity.
\end{abstract}

Keywords: Vlasov-like equations; measure solutions; swarming; Cucker-Smale model; Vicsek model; Laplace-Beltrami operator; generalized collision invariants.

AMS Subject Classification: 92D50, 82C40, 92C10

* Corresponding author

This is an Open Access article published by World Scientific Publishing Company. It is distributed under the terms of the Creative Commons Attribution 4.0 (CC-BY) License. Further distribution of this work is permitted, provided the original work is properly cited. 


\section{Introduction}

The subject matter of this paper concerns the behavior of living organisms such as flocks of birds, school of fish, swarms of insects, myxobacteria, etc. These models include short-range repulsion, long-range attraction, self-propelling and friction forces, reorientation or alignment (see Refs. 4, 65, 58, 66, 60, 53, 36, 57, 7 and 6. We consider self-propelled particles with Rayleigh friction, and alignment, introduced through the Cucker-Smale reorientation procedure, 38 , see also Refs. 56, 55, 28, 29, 61 and 62 for further details and Ref. 59 for a survey. If we denote by $f=f(t, x, v) \geq 0$ the particle density in the phase space $(x, v) \in \mathbb{R}^{d} \times \mathbb{R}^{d}$, with $d \in\{2,3\}$, the self-propulsion/friction mechanism is given by the term $\operatorname{div}_{v}\left\{f\left(\alpha-\beta|v|^{2}\right) v\right\}$. Notice that the balance between the self-propulsion and friction forces occurs on the velocity sphere $|v|=r:=\sqrt{\alpha / \beta}$. We fix the speed $r$, meaning that $\alpha$ and $\beta$ are anytime related by the equality $\alpha=\beta r^{2}$. The coefficients $\alpha, \beta>0$ can be interpreted as follows. In the absence of friction, the particles accelerate with $\alpha v$, leading to an exponential growth of velocity, with frequency $\alpha$. In the absence of self-propulsion, the inverse of the relative kinetic energy grows linearly, with the frequency $2 \beta|v|^{2}$, where $v$ is the initial velocity of the particle

$$
\frac{\mathrm{d}}{\mathrm{d} s} \frac{|v|^{2}}{|V(s)|^{2}}=-\frac{|v|^{2}}{|V(s)|^{4}} 2\left(V(s) \cdot V^{\prime}(s)\right)=2 \beta|v|^{2} .
$$

Each individual in the group relaxes its velocity toward the mean velocity of the neighbors, leading to the term $\nu \operatorname{div}_{v}\{f(u[f]-v)\}$, where $\nu$ is the reorientation frequency and $u[f]$ is the mean velocity

$$
u[f(t)](x)=\frac{\int_{\mathbb{R}^{d}} \int_{\mathbb{R}^{d}} f\left(t, x^{\prime}, v^{\prime}\right) h\left(x-x^{\prime}\right) v^{\prime} \mathrm{d} v^{\prime} \mathrm{d} x^{\prime}}{\int_{\mathbb{R}^{d}} \int_{\mathbb{R}^{d}} f\left(t, x^{\prime}, v^{\prime}\right) h\left(x-x^{\prime}\right) \mathrm{d} v^{\prime} \mathrm{d} x^{\prime}} .
$$

The weight application $h$ is a decreasing, radial, non-negative given function that determines the interaction neighborhood around any position. By including also noise in the above kinetic model, we get to the Fokker-Planck-like equation

$$
\begin{aligned}
\partial_{t} f & +\operatorname{div}_{x}(f v)+\operatorname{div}_{v}\left\{f\left(\alpha-\beta|v|^{2}\right) v\right\} \\
& =\nu \operatorname{div}_{v}\{f(v-u[f])\}+\tau \Delta_{v} f \\
& =\nu \operatorname{div}_{v}\left\{f(v-u[f])+\sigma \nabla_{v} f\right\}:=\nu Q(f),
\end{aligned}
$$

where $\sigma=\tau / \nu$ represents the diffusion coefficient in the velocity space. We investigate the large time and space scale regime of (1.1) that is, we fix large time and space units. In this case, Eq. (1.1) should be replaced by

$$
\varepsilon_{1}\left\{\partial_{t} f+\operatorname{div}_{x}(f v)\right\}+\operatorname{div}_{v}\left\{f\left(\alpha-\beta|v|^{2}\right) v\right\}=\nu Q(f) .
$$

The choice of a large length unit leads to a local reorientation mechanism: the mean velocity $u[f]$ in (1.2) is now given by

$$
u[f(t)](x)=\frac{\int_{\mathbb{R}^{d}} f\left(t, x, v^{\prime}\right) v^{\prime} \mathrm{d} v^{\prime}}{\int_{\mathbb{R}^{d}} f\left(t, x, v^{\prime}\right) \mathrm{d} v^{\prime}} .
$$


Notice that if $f(t, x, \cdot)=0$, then the Fokker-Planck collision operator vanishes for any $u$. In this case we can define $u[f(t)]=0$, without loss of generality. We assume that the frequencies $\varepsilon_{1}$ and $\nu$ scale like $\frac{\nu}{\varepsilon_{1}} \approx \frac{1}{\varepsilon_{2}}$ for some small parameters $\varepsilon_{1}, \varepsilon_{2}>0$ and thus Eq. (1.2) becomes

$$
\partial_{t} f^{\varepsilon_{1}, \varepsilon_{2}}+\operatorname{div}_{x}\left(f^{\varepsilon_{1}, \varepsilon_{2}} v\right)+\frac{1}{\varepsilon_{1}} \operatorname{div}_{v}\left\{f^{\varepsilon_{1}, \varepsilon_{2}}\left(\alpha-\beta|v|^{2}\right) v\right\}=\frac{1}{\varepsilon_{2}} Q\left(f^{\varepsilon_{1}, \varepsilon_{2}}\right) .
$$

Assume for the moment that $\varepsilon_{1} \searrow 0$ and $\varepsilon_{2}$ is fixed. In this situation, the leading order term in the Fokker-Planck equation (1.3) corresponds to the self-propulsion/friction mechanism, and we expect that the limit density $f^{\varepsilon_{2}}=$ $\lim _{\varepsilon_{1} \searrow 0} f^{\varepsilon_{1}, \varepsilon_{2}}$ satisfies

$$
\operatorname{div}_{v}\left\{f^{\varepsilon_{2}}\left(\alpha-\beta|v|^{2}\right) v\right\}=0 .
$$

The previous constraint exactly says that at any time $t$ and any position $x$, the velocity distribution $f^{\varepsilon_{2}}(t, x, \cdot)$ is a measure supported in $\{0\} \cup r \mathbb{S}^{d-1}$ cf. Ref. 20. The particles will tend to move with asymptotic speed $r$. These models have been shown to produce complicated dynamics and patterns at the particle level such as mills, double mills, flocks and clumps, see Ref. [50, whose stability properties are very relevant in the applications, see Refs. 8, 3 and 31. Assuming that all individuals move with constant speed also leads to spatial aggregation, patterns, and collective motion.40|51/64 More exactly, it was shown in Ref. 20 that, by taking the limit $\varepsilon_{1} \searrow 0$, the solutions $f^{\varepsilon_{1}, \varepsilon_{2}}$ of (1.3) converge toward the solution $f^{\varepsilon_{2}}$ of

$$
\partial_{t} f^{\varepsilon_{2}}+\operatorname{div}_{x}\left(f^{\varepsilon_{2}} \omega\right)+\frac{1}{\varepsilon_{2}} \operatorname{div}_{\omega}\left\{f^{\varepsilon_{2}}\left(I_{d}-\frac{\omega \otimes \omega}{r^{2}}\right) u\left[f^{\varepsilon_{2}}\right]\right\}=\frac{\sigma}{\varepsilon_{2}} \Delta_{\omega} f^{\varepsilon_{2}},
$$

for all $(t, x, \omega) \in \mathbb{R}_{+} \times \mathbb{R}^{d} \times r \mathbb{S}^{d-1}$ with

$$
u\left[f^{\varepsilon_{2}}(t)\right](x)=\frac{\int_{r \mathbb{S}^{d-1}} f^{\varepsilon_{2}}(t, x, \omega) \omega \mathrm{d} \omega}{\int_{r \mathbb{S}^{d-1}} f^{\varepsilon_{2}}(t, x, \omega) \mathrm{d} \omega}, \quad(t, x) \in \mathbb{R}_{+} \times \mathbb{R}^{d} .
$$

The above result states that in the limit $\varepsilon_{1} \searrow 0$, the Cucker-Smale model with diffusion is reduced to a Vicsek-like model, whose phase transition was analyzed in Ref.52. The evolution problem (1.4) on the phase space $\mathbb{R}^{d} \times r \mathbb{S}^{d-1}$, with normalized velocity field $u\left[f^{\varepsilon_{2}}\right]$, i.e.

$$
\partial_{t} f+\operatorname{div}_{x}(f \omega)+\nu \operatorname{div}_{\omega}\left\{f\left(I_{d}-\frac{\omega \otimes \omega}{r^{2}}\right) \Omega[f]\right\}=\tau \Delta_{\omega} f
$$

for all $(t, x, \omega) \in \mathbb{R}_{+} \times \mathbb{R}^{d} \times r \mathbb{S}^{d-1}$ with

$$
\Omega[f(t)](x)=\frac{\int_{r \mathbb{S}^{d-1}} f(t, x, \omega) \omega \mathrm{d} \omega}{\left|\int_{r \mathbb{S}^{d-1}} f(t, x, \omega) \omega \mathrm{d} \omega\right|}, \quad(t, x) \in \mathbb{R}_{+} \times \mathbb{R}^{d}
$$

was also proposed in the literature as continuum version 48 of the Vicsek model. 66.36 Furthermore, the full phase transition for stationary solutions and their asymptotic stability was subsequently generalized in Refs. 41 and 42 allowing for quite general dependency of $\nu$ and $\tau$ on $|u[f(t)]|$. We will focus on the relaxation toward the mean 
velocity $u[f]$, whose alignment mechanism relies only on the direction of the mean velocity $\Omega[f]=u[f] /|u[f]|$. Nevertheless, our method still applies and allows us to handle the model with normalization and the generalizations in Refs. 48 and 42 as well.

The original kinetic Vicsek model in Refs. 66] and 37 was derived as the meanfield limit of some stochastic particle systems in Ref. 10. In fact, previous particle systems have also been studied with noise in Ref. 9 for the mean-field limit (see also Refs. 63, 21, 49, 23, 2, 24, 26), in Ref. 54 for studying some properties of the Cucker-Smale model with noise, and in Refs. 5 and 33 for phase transitions at the level of the Cucker-Smale model and the inhomogeneous level, respectively.

We assume now that both $\varepsilon_{1}, \varepsilon_{2}$ become small. The idea is to justify a macroscopic model for (1.4), resulting from the balance between two opposite phenomena:

(1) The reorientation, which tends to align the particle velocities with respect to the mean velocity;

(2) The diffusion, which tends to spread the particle velocities isotropically on the sphere $r \mathbb{S}^{d-1}$.

Such hydrodynamic models were obtained in Refs. 48 and 42 by letting $\varepsilon_{2} \searrow 0$ in the normalized alignment version of (1.4). They are typically referred as selforganized hydrodynamics $(\mathrm{SOH})$. Notice that the $\mathrm{SOH}$ model was obtained by passing to the limit successively in (1.3) with respect to $\varepsilon_{1}, \varepsilon_{2}$. After letting $\varepsilon_{1} \searrow 0$, the dynamics were reduced to the phase space $(x, v) \in \mathbb{R}^{d} \times r \mathbb{S}^{d-1}$, but still captures microscopic behavior in the tangent directions to the sphere $r \mathbb{S}^{d-1}$. The second limit procedure, $\varepsilon_{2} \searrow 0$, leads to the macroscopic equations for the density $\int_{r \mathbb{S}^{d-1}} f \mathrm{~d} \omega$ and the direction of the flux $\int_{r \mathbb{S}^{d-1}} \omega f \mathrm{~d} \omega$.

We intend to obtain a SOH model, by passing to the limit in (1.3), simultaneously with respect to $\left(\varepsilon_{1}, \varepsilon_{2}\right)$. Motivated by the above discussion, we assume that $\varepsilon_{1}=\varepsilon^{2}$ and $\varepsilon_{2}=\varepsilon$, where $\varepsilon>0$ is a small parameter, that is, the selfpropulsion/friction mechanism dominates the alignment. This implies that $\nu=\varepsilon$ and $\tau=\sigma \varepsilon$. Therefore (1.3) becomes

$$
\partial_{t} f^{\varepsilon}+\operatorname{div}_{x}\left(f^{\varepsilon} v\right)+\frac{1}{\varepsilon^{2}} \operatorname{div}_{v}\left\{f^{\varepsilon}\left(\alpha-\beta|v|^{2}\right) v\right\}=\frac{1}{\epsilon} Q(f),
$$

for all $(t, x, v) \in \mathbb{R}_{+} \times \mathbb{R}^{2 d}$, supplemented by the initial condition

$$
f^{\varepsilon}(0, x, v)=f^{\text {in }}(x, v), \quad(x, v) \in \mathbb{R}^{d} \times \mathbb{R}^{d} .
$$

Very recently, by a similar scaling, fluid models have been obtained for the transport of charged particles, under the action of strong magnetic fields, which dominate the collision effects. The resulting macroscopic model is a gyrokinetic version of the Euler equations, in the parallel direction with respect to the magnetic field! 15,16

The behavior of the family $\left(f^{\varepsilon}\right)_{\varepsilon>0}$, as the parameter $\varepsilon$ becomes small, follows by analyzing the formal expansion

$$
f^{\varepsilon}=f+\varepsilon f^{(1)}+\varepsilon^{2} f^{(2)}+\cdots .
$$


Plugging the above ansatz into (1.5) leads to the constraints

$$
\begin{aligned}
\operatorname{div}_{v}\left\{f\left(\alpha-\beta|v|^{2}\right) v\right\} & =0, \\
\operatorname{div}_{v}\left\{f^{(1)}\left(\alpha-\beta|v|^{2}\right) v\right\} & =\operatorname{div}_{v}\left\{f(v-u[f])+\sigma \nabla_{v} f\right\}
\end{aligned}
$$

and to the time evolution equations

$$
\partial_{t} f+\operatorname{div}_{x}(f v)+\operatorname{div}_{v}\left\{f^{(2)}\left(\alpha-\beta|v|^{2}\right) v\right\}=\mathcal{L}_{f}\left(f^{(1)}\right)
$$

with

$$
\mathcal{L}_{f}\left(f^{(1)}\right):=\operatorname{div}_{v}\left\{f^{(1)}(v-u[f])+\sigma \nabla_{v} f^{(1)}\right\}-\operatorname{div}_{v}\left\{f \frac{\int_{\mathbb{R}^{d}} f^{(1)}\left(v^{\prime}-u[f]\right) \mathrm{d} v^{\prime}}{\int_{\mathbb{R}^{d}} f \mathrm{~d} v^{\prime}}\right\}
$$

cutting the development at second order.

We expect the same macroscopic SOH model for the moments of $f$ as obtained in Refs. 48, 41 and 42. The main advantage for considering (1.5) instead of (1.4) with $\varepsilon_{2}=\varepsilon$ is that the resolution of (1.5) for small $\varepsilon$ will provide a solution supported near $\mathbb{R}^{d} \times r \mathbb{S}^{d-1}$, which fits much better the behavior of living organism systems, than the solution of 1.4 on $\mathbb{R}^{d} \times r \mathbb{S}^{d-1}$. But the price to pay is to deal with two Lagrange multipliers, appearing in (1.9), which have to be eliminated, thanks to the constraints (1.7) and (1.8). The first constraint was analyzed in detail in Ref. 20. It exactly says that $f$ is a measure supported in $\mathbb{R}^{d} \times\left(\{0\} \cup r \mathbb{S}^{d-1}\right)$. We denote by $\mathcal{M}_{b}^{+}\left(\mathbb{R}^{d}\right)$ the set of non-negative bounded Radon measure on $\mathbb{R}^{d}$.

Proposition 1.1. Assume that $\left(1+|v|^{2}\right) F \in \mathcal{M}_{b}^{+}\left(\mathbb{R}^{d}\right)$. Then $F$ solves $\operatorname{div}_{v}\{F(\alpha-$ $\left.\left.\beta|v|^{2}\right) v\right\}=0$ in $\mathcal{D}^{\prime}\left(\mathbb{R}^{d}\right)$, i.e.

$$
\int_{\mathbb{R}^{d}}\left(\alpha-\beta|v|^{2}\right) v \cdot \nabla_{v} \varphi \mathrm{d} F(v)=0, \quad \text { for any } \varphi \in C_{c}^{1}\left(\mathbb{R}^{d}\right)
$$

if and only if $\operatorname{supp} F \subset\{0\} \cup r \mathbb{S}^{d-1}$.

The proof of Proposition 1.1 is based on the resolution of the adjoint problem

$$
-\left(\alpha-\beta|v|^{2}\right) v \cdot \nabla_{v} \varphi=\psi(v), \quad v \in \mathbb{R}^{d},
$$

for any smooth function $\psi$ with compact support in $\mathbb{R}^{d} \backslash\left(\{0\} \cup r \mathbb{S}^{d-1}\right)$, cf. Lemma 3.1 of Ref. 20,

Lemma 1.1. For any $C^{1}$-function $\psi=\psi(v)$ with compact support in $\mathbb{R}^{d} \backslash(\{0\} \cup$ $\left.r \mathbb{S}^{d-1}\right)$, there is a bounded $C^{1}$-function $\varphi=\varphi(v)$ such that $\varphi(0)=0$ and

$$
-\left(\alpha-\beta|v|^{2}\right) v \cdot \nabla_{v} \varphi=\psi(v), \quad v \in \mathbb{R}^{d} .
$$

In the sequel, we introduce a projection operator onto the subspace of the constraints in (1.7). This construction follows closely the gyroaverage method in gyrokinetic theory!11,14,17,19 An average operator serves to separate between two scales. For example, in gyrokinetic theory, two time scales exist: a fast time 
variable, related to the rapid cyclotronic motion, and a slow time variable, related to the parallel motion with respect to the magnetic field. The gyroaverage operator represents the average of the fast dynamics over a cyclotronic period, provided that the slow time variable is frozen. Following this technique, we obtain an accurate enough but simpler model, from the numerical approximation point of view. All the fluctuations have been removed and replaced by averaged effects.

Our model (1.5) presents not two, but three time variables: $t, t / \varepsilon$ and $t / \varepsilon^{2}$. The dynamics are dominated by the self-propulsion/friction mechanism, introducing the fast time variable $s=t / \varepsilon^{2}$. The average operator is related to the characteristic flow of the field $\frac{1}{\varepsilon^{2}}\left(\alpha-\beta|v|^{2}\right) v \cdot \nabla_{v}$. This characteristic flow $\mathcal{V}=\mathcal{V}(s ; v)$, written with respect to $s=t / \varepsilon^{2}$ :

$$
\frac{\mathrm{d} \mathcal{V}}{\mathrm{d} s}=\left(\alpha-\beta|\mathcal{V}(s ; v)|^{2}\right) \mathcal{V}(s ; v), \quad \mathcal{V}(0 ; v)=v
$$

conserves the direction $\frac{v}{|v|}$ and has as equilibria the elements of $\{0\} \cup r \mathbb{S}^{d-1}$. The Jacobian matrix is given by

$$
\partial_{v}\left\{\left(\alpha-\beta|v|^{2}\right) v\right\}=\left(\alpha-\beta|v|^{2}\right) I_{d}-2 \beta v \otimes v .
$$

Being negative on $r \mathbb{S}^{d-1}$ and definite positive at 0 , we deduce that the points of $r \mathbb{S}^{d-1}$ are stable equilibria, and 0 is an unstable equilibrium. For simplicity, we neglect the measure of the unstable point 0 in the velocity space and assume that this is not present in the limit $\epsilon \rightarrow 0$ at any level of the expansion. As we elaborate below, we will rigorously compute the terms in the expansion needed to derive formally the hydrodynamic equations. The complete mathematical analysis of the limiting procedure is out of scope of this paper. We are mainly interested in the two- or three-dimensional setting, but the same arguments apply for any dimension $d \geq 2$. For the sake of generality, we state and prove all the results in any dimension $d \geq 2$, and we distinguish, if necessary, between the cases $d=2$ and $d \geq 3$.

Motivated by the previous observations, we define the average of a non-negative bounded measure, cf. Ref. 20] We will denote by $f(x, v) \mathrm{d} v \mathrm{~d} x$ the integration against the measure $f$. This is done independently of being the measure $f$ absolutely continuous with respect to the Lebesgue measure or not.

Definition 1.1. (1) Let $F \in \mathcal{M}_{b}^{+}\left(\mathbb{R}^{d}\right)$ be a non-negative bounded measure on $\mathbb{R}^{d}$. We denote by $\langle F\rangle$ the measure corresponding to the linear application

$$
\psi \rightarrow \int_{\mathbb{R}^{d}} \psi(v) \mathbf{1}_{v=0} F(v) \mathrm{d} v+\int_{\mathbb{R}^{d}} \psi\left(r \frac{v}{|v|}\right) \mathbf{1}_{v \neq 0} F(v) \mathrm{d} v,
$$

for all $\psi \in C_{c}^{0}\left(\mathbb{R}^{d}\right)$, i.e.

$$
\int_{\mathbb{R}^{d}} \psi(v)\langle F\rangle(v) \mathrm{d} v=\int_{v=0} \psi(v) F(v) \mathrm{d} v+\int_{v \neq 0} \psi\left(r \frac{v}{|v|}\right) F(v) \mathrm{d} v,
$$

for all $\psi \in C_{c}^{0}\left(\mathbb{R}^{d}\right)$. 
(2) Let $f \in \mathcal{M}_{b}^{+}\left(\mathbb{R}^{d} \times \mathbb{R}^{d}\right)$ be a non-negative bounded measure on $\mathbb{R}^{d} \times \mathbb{R}^{d}$. We denote by $\langle f\rangle$ the measure corresponding to the linear application

$$
\psi \rightarrow \int_{\mathbb{R}^{d}} \int_{\mathbb{R}^{d}} \psi(x, v) \mathbf{1}_{v=0} f(x, v) \mathrm{d} v \mathrm{~d} x+\int_{\mathbb{R}^{d}} \int_{\mathbb{R}^{d}} \psi\left(x, r \frac{v}{|v|}\right) \mathbf{1}_{v \neq 0} f(x, v) \mathrm{d} v \mathrm{~d} x,
$$

for all $\psi \in C_{c}^{0}\left(\mathbb{R}^{d} \times \mathbb{R}^{d}\right)$, i.e.

$$
\begin{aligned}
\int_{\mathbb{R}^{d}} \int_{\mathbb{R}^{d}} \psi(x, v)\langle f\rangle(x, v) \mathrm{d} v \mathrm{~d} x= & \int_{v=0} \psi(x, v) f(x, v) \mathrm{d} v \mathrm{~d} x \\
& +\int_{v \neq 0} \psi\left(x, r \frac{v}{|v|}\right) f(x, v) \mathrm{d} v \mathrm{~d} x
\end{aligned}
$$

for all $\psi \in C_{c}^{0}\left(\mathbb{R}^{d} \times \mathbb{R}^{d}\right)$.

It is easily seen that the average of a non-negative bounded measure is a nonnegative bounded measure, with the same mass, but supported in $\{0\} \cup r \mathbb{S}^{d-1}$, $\mathbb{R}^{d} \times\left(\{0\} \cup r \mathbb{S}^{d-1}\right)$, respectively. We have the following characterization (see Proposition 5.1 of Ref. (20).

Proposition 1.2. Assume that $f$ is a non-negative bounded measure on $\mathbb{R}^{d} \times \mathbb{R}^{d}$. Then $\langle f\rangle$ is the unique measure $\tilde{f}$ satisfying $\operatorname{supp} \tilde{f} \subset \mathbb{R}^{d} \times\left(\{0\} \cup r \mathbb{S}^{d-1}\right)$,

$\int_{v \neq 0} \psi\left(x, r \frac{v}{|v|}\right) \tilde{f}(x, v) \mathrm{d} v \mathrm{~d} x=\int_{v \neq 0} \psi\left(x, r \frac{v}{|v|}\right) f(x, v) \mathrm{d} v \mathrm{~d} x, \quad \psi \in C_{c}^{0}\left(\mathbb{R}^{d} \times \mathbb{R}^{d}\right)$ and $\tilde{f}=f$ on $\mathbb{R}^{d} \times\{0\}$.

A direct consequence of Proposition 1.2 is that any bounded, non-negative measure, supported in $\mathbb{R}^{d} \times\left(\{0\} \cup r \mathbb{S}^{d-1}\right)$ is left unchanged by the average operator. Another property of the average operator is that it removes any measure of the form $\operatorname{div}_{v}\left\{f\left(\alpha-\beta|v|^{2}\right) v\right\}$, cf. Proposition 5.2 of Ref. [20.

Proposition 1.3. For any $f \in \mathcal{M}_{b}^{+}\left(\mathbb{R}^{d} \times \mathbb{R}^{d}\right)$ such that $\operatorname{div}_{v}\left\{f\left(\alpha-\beta|v|^{2}\right) v\right\} \in$ $\mathcal{M}_{b}\left(\mathbb{R}^{d} \times \mathbb{R}^{d}\right)$, we have $\left\langle\operatorname{div}_{v}\left\{f\left(\alpha-\beta|v|^{2}\right) v\right\}\right\rangle=0$.

The above proposition plays a crucial role when eliminating the Lagrange multiplier $f^{(2)}$ in (1.9). Indeed, for doing that, it is enough to average both hand sides in (1.9). By the constraint (1.7), we know that $f$ is supported in $\mathbb{R}^{d} \times\left(\{0\} \cup r \mathbb{S}^{d-1}\right)$, and thus is left invariant by the average. We check that $\left\langle\partial_{t} f\right\rangle=\partial_{t}\langle f\rangle=\partial_{t} f$, and thus, averaging (1.9) still leads to an evolution problem for $f$ :

$$
\partial_{t} f+\left\langle\operatorname{div}_{x}(f v)\right\rangle=\left\langle\mathcal{L}_{f}\left(f^{(1)}\right)\right\rangle .
$$

Certainly, a much more difficult task is to eliminate the Lagrange multiplier $f^{(1)}$. We expect that this can be done thanks to the constraint in (1.8). The solvability 
of (1.8), with respect to $f^{(1)}$, depends on a compatibility condition, to be satisfied by the right-hand side. Indeed, by Proposition [1.3. we should have

$$
\left\langle\operatorname{div}_{v}\left\{f(v-u[f])+\sigma \nabla_{v} f\right\}\right\rangle=\left\langle\operatorname{div}_{v}\left\{f^{(1)}\left(\alpha-\beta|v|^{2}\right) v\right\}\right\rangle=0,
$$

saying that $f$ is an equilibrium for the average collision kernel $\langle Q(f)\rangle=0$. The equilibria of the average collision kernel form a $(d-1)$-dimensional manifold, that is one dimension less than the equilibria manifold of the Fokker-Planck operator $Q$ (see also Refs. 48 and [52). For any $l \in \mathbb{R}_{+}, \Omega \in \mathbb{S}^{d-1}$, we introduce the von Mises-Fisher distribution

$$
M_{l \Omega}(\omega) \mathrm{d} \omega=\frac{\exp \left(l \Omega \cdot \frac{\omega}{r}\right)}{\int_{r \mathbb{S}^{d-1}} \exp \left(l \Omega \cdot \frac{\omega^{\prime}}{r}\right) \mathrm{d} \omega^{\prime}} \mathrm{d} \omega, \quad \omega \in r \mathbb{S}^{d-1} .
$$

Proposition 1.4. Let $F \in \mathcal{M}_{b}^{+}\left(\mathbb{R}^{d}\right)$ be a non-negative bounded measure on $\mathbb{R}^{d}$, supported in $r \mathbb{S}^{d-1}$. The following statements are equivalent:

(1) $\langle Q(F)\rangle=0$, that is

$$
\int_{v \neq 0}\left\{-(v-u[F]) \cdot \nabla_{v}\left[\widetilde{\psi}\left(r \frac{v}{|v|}\right)\right]+\sigma \Delta_{v}\left[\widetilde{\psi}\left(r \frac{v}{|v|}\right)\right]\right\} F \mathrm{~d} v=0,
$$

for all $\widetilde{\psi} \in C^{2}\left(r \mathbb{S}^{d-1}\right)$.

(2) There are $\rho \in \mathbb{R}_{+}, \Omega \in \mathbb{S}^{d-1}$ such that $F=\rho M_{l \Omega} \mathrm{d} \omega$ where $l \in \mathbb{R}_{+}$satisfies

$$
\frac{\int_{0}^{\pi} \cos \theta e^{l \cos \theta} \sin ^{d-2} \theta \mathrm{d} \theta}{\int_{0}^{\pi} e^{l \cos \theta} \sin ^{d-2} \theta \mathrm{d} \theta}=\frac{\sigma}{r^{2}} l .
$$

The modulus of the mean velocity is not a coordinate on the equilibria manifold, but it is determined by the condition $|u|=\frac{\sigma l}{r}$ where $l$ satisfies (1.11). Clearly $l=0$ is a solution, which corresponds to the isotropic equilibrium

$$
F=\rho M_{0 \Omega} \mathrm{d} \omega=\rho \frac{\mathrm{d} \omega}{\bar{\omega}_{d} r^{d-1}},
$$

where $\bar{\omega}_{d}$ represents the area of the unit sphere in $\mathbb{R}^{d}$. The next proposition is essentially contained in Proposition 3.3 in Ref. 52 We present a simplified proof, based on computations with Bessel functions.

Proposition 1.5. Let $\lambda: \mathbb{R}_{+} \rightarrow \mathbb{R}$ be the function given by

$$
\lambda(l)=\frac{\int_{0}^{\pi} \cos \theta e^{l \cos \theta} \sin ^{d-2} \theta \mathrm{d} \theta}{\int_{0}^{\pi} e^{l \cos \theta} \sin ^{d-2} \theta \mathrm{d} \theta}, \quad l \in \mathbb{R}_{+}, \quad d \geq 2 .
$$

The function $\lambda$ is strictly increasing, the function $l \rightarrow \lambda(l) / l$ is strictly decreasing and verifies

$$
\lambda(0)=0, \quad \lambda^{\prime}(0)=\lim _{l \searrow 0} \frac{\lambda(l)}{l}=\frac{1}{d}, \quad \lim _{l \rightarrow+\infty} \lambda(l)=1 .
$$

If $\frac{\sigma}{r^{2}} \geq \frac{1}{d}$, then the only solution of $\lambda(l)=\frac{\sigma}{r^{2}} l$ is $l=0$. If $\left.\frac{\sigma}{r^{2}} \in\right] 0, \frac{1}{d}[$, then there is a unique $l=l\left(\frac{\sigma}{r^{2}}\right)>0$ such that $\lambda(l)=\frac{\sigma}{r^{2}} l$. 
In order to find the equations for the evolution of the density $\rho$ and orientation $\Omega$, we need to find $f^{(1)}$ from (1.8) in order to feed the terms needed in (1.10). However, we will see that this is not possible. We will need to introduce a notion of generalized collision invariants, quite related intuitively to the one introduced in Refs. 48, 41 and 42, in our functional setting of measures supported in $r \mathbb{S}^{d-1}$ to avoid the computation of the full $f^{(1)}$. This is the main technical difficulty due to the measure functional setting since the precise definition of generalized collision invariant we need is more involved than in Refs. 48, 41 and 42. Let us mention that this notion of generalized collision invariant has been used in other related models in collective dynamics $47 / 43,44$ and in kinetic models of wealth distribution. 16

Our main result establishes the macroscopic equations satisfied by the density $\rho$ and orientation $\Omega$, which parametrize the von Mises-Fisher equilibrium, obtained when passing to the limit for $\varepsilon \searrow 0$ in (1.5). We retrieve exactly the limit $\mathrm{SOH}$ hydrodynamic model in Ref. 41, written for any space dimension $d \geq 2$ with the same explicit constants.

Theorem 1.1. For any $\sigma, r$ such that $\left.\frac{\sigma}{r^{2}} \in\right] 0, \frac{1}{d}\left[\right.$, we denote by $l=l\left(\frac{\sigma}{r^{2}}\right)$ the unique positive solution of $\lambda(l)=\frac{\sigma}{r^{2}} l$. Let $f^{\text {in }} \in \mathcal{M}_{b}^{+}\left(\mathbb{R}^{d} \times \mathbb{R}^{d}\right)$ be a non-negative bounded measure on $\mathbb{R}^{d} \times \mathbb{R}^{d}, d \geq 2$. For any $\varepsilon>0$ we consider the problem

$$
\partial_{t} f^{\varepsilon}+\operatorname{div}_{x}\left(f^{\varepsilon} v\right)+\frac{1}{\varepsilon^{2}} \operatorname{div}_{v}\left(f^{\varepsilon}\left(\alpha-\beta|v|^{2}\right) v\right)=\frac{1}{\varepsilon} \operatorname{div}_{v}\left\{f^{\varepsilon}\left(v-u\left[f^{\varepsilon}\right]\right)+\sigma \nabla_{v} f^{\varepsilon}\right\},
$$

for all $(t, x, v) \in \mathbb{R}_{+} \times \mathbb{R}^{d} \times \mathbb{R}^{d}$ with $f^{\varepsilon}(0)=f^{\text {in }},(x, v) \in \mathbb{R}^{d} \times \mathbb{R}^{d}$. Therefore the limit distribution $f=\lim _{\varepsilon \backslash 0} f^{\varepsilon}$ is a von Mises-Fisher equilibrium $f=\rho M_{l \Omega}(\omega) \mathrm{d} \omega$ on $r \mathbb{S}^{d-1}$, where the density $\rho(t, x)$ and the orientation $\Omega(t, x)$ satisfy the macroscopic equations:

$$
\begin{gathered}
\partial_{t} \rho+\operatorname{div}_{x}\left(\rho \frac{l \sigma}{r} \Omega\right)=0, \quad(t, x) \in \mathbb{R}_{+} \times \mathbb{R}^{d}, \\
\partial_{t} \Omega+k_{d} r\left(\Omega \cdot \nabla_{x}\right) \Omega+\frac{r}{l}\left(I_{d}-\Omega \otimes \Omega\right) \frac{\nabla_{x} \rho}{\rho}=0,
\end{gathered}
$$

with the initial conditions

$$
\rho(0, x)=\int_{\mathbb{R}^{d}} f^{\text {in }}(x) \mathrm{d} v, \quad \Omega(0, x)=\frac{\int_{\mathbb{R}^{d}} v f^{\text {in }}(x) \mathrm{d} v}{\left|\int_{\mathbb{R}^{d}} v f^{\text {in }}(x) \mathrm{d} v\right|}, \quad x \in \mathbb{R}^{d},
$$

where

$$
k_{d}=\frac{\int_{0}^{\pi} e^{l \cos \theta} \chi(\cos \theta) \cos \theta \sin ^{d-1} \theta \mathrm{d} \theta}{\int_{0}^{\pi} e^{l \cos \theta} \chi(\cos \theta) \sin ^{d-1} \theta \mathrm{d} \theta}
$$

and $\chi$ solves

$$
\left.-\frac{\sigma}{r^{2}} \frac{\mathrm{d}}{\mathrm{d} c}\left\{e^{l c} \chi^{\prime}(c)\left(1-c^{2}\right)^{\frac{1}{2}}\right\}=r e^{l c}, \quad c \in\right]-1,1[, \quad \chi(-1)=\chi(1)=0 \quad \text { if } d=2
$$


and

$$
\begin{aligned}
& -\frac{\sigma}{r^{2}} \frac{\mathrm{d}}{\mathrm{d} c}\left\{e^{l c} \chi^{\prime}(c)\left(1-c^{2}\right)^{\frac{d-1}{2}}\right\}+(d-2) \frac{\sigma}{r^{2}} e^{l c} \chi(c)\left(1-c^{2}\right)^{\frac{d-5}{2}}=r e^{l c}\left(1-c^{2}\right)^{\frac{d-2}{2}}, \\
& c \in]-1,1[, d \geq 3 \text {. }
\end{aligned}
$$

A nice practical implication of our main result is that this penalization procedure, by imposing asymptotically a cruise speed for particles, could lead to efficient and stable numerical schemes to compute the hydrodynamic equations (1.13)-(1.14). This is important due to the possible non-hyperbolicity of the system 1.13)-1.14, see Ref. 42. The local in time well-posedness of the SOH system 1.13 - 1.14) was studied in Ref. 45. We finally emphasize that the constants appearing in Eqs. (1.13)-(1.14) coincide exactly with the ones obtained in Ref. 42 after some easy but tedious algebraic manipulations.

Our paper is organized as follows. In Sec. 2, we study the equilibria of the average collision operator in our functional setting. This analysis can be carried out by introducing some Bessel functions. In the next section we investigate the notion of collision invariant suitable in our functional setting. We determine the structure of these invariants and present their symmetries. Section 4 is devoted to the derivation of the fluid model for the macroscopic quantities, parametrizing the limit von Mises-Fisher equilibrium. The proofs of some technical results can be found in Appendices $\mathrm{A}-\mathrm{C}$.

\section{The Equilibria of the Average Collision Operator}

We consider the collision operator $Q(F)=\operatorname{div}_{v}\left\{F(v-u[F])+\sigma \nabla_{v} F\right\}$ where $u[F]=$ $\int_{\mathbb{R}^{d}} v F \mathrm{~d} v / \int_{\mathbb{R}^{d}} F \mathrm{~d} v$ is the mean velocity. The above operator should be understood in the duality sense between non-negative bounded measures on $\mathbb{R}^{d}$ and smooth functions, compactly supported in $\mathbb{R}^{d}$ :

$$
\int_{\mathbb{R}^{d}} \psi(v) Q(F) \mathrm{d} v=\int_{\mathbb{R}^{d}}\left[-(v-u[F]) \cdot \nabla_{v} \psi(v)+\sigma \Delta_{v} \psi(v)\right] F \mathrm{~d} v,
$$

for any $F \in \mathcal{M}_{b}^{+}\left(\mathbb{R}^{d}\right)$ and $\psi \in C_{c}^{2}\left(\mathbb{R}^{d}\right)$ such that $\int_{\mathbb{R}^{d}}|v| F \mathrm{~d} v<+\infty$. As suggested by the formal expansion (1.6), we focus on measures satisfying (see (1.7)-(11.8)):

$$
\operatorname{div}_{v}\left\{F\left(\alpha-\beta|v|^{2}\right) v\right\}=0, \quad Q(F)=\operatorname{div}_{v}\left\{F^{(1)}\left(\alpha-\beta|v|^{2}\right) v\right\} .
$$

Thanks to Propositions 1.3 and 1.1, we deduce that $\operatorname{supp} F \subset\{0\} \cup r \mathbb{S}^{d-1}$ and

$$
\langle Q(F)\rangle=\left\langle\operatorname{div}_{v}\left\{F^{(1)}\left(\alpha-\beta|v|^{2}\right) v\right\}\right\rangle=0 .
$$

We discuss the case of non-negative bounded measures supported on the sphere $r \mathbb{S}^{d-1}$, that is, we discard all difficulties related to the mass of the points at rest. For such measures, the equality $\langle Q(F)\rangle=0$ can be interpreted in the following 
sense (see Proposition 1.2):

$$
\int_{v \neq 0}\left\{-(v-u[F]) \cdot \nabla_{v}\left[\widetilde{\psi}\left(r \frac{v}{|v|}\right)\right]+\sigma \Delta_{v}\left[\widetilde{\psi}\left(r \frac{v}{|v|}\right)\right]\right\}=0, \quad \forall \widetilde{\psi} \in C^{2}\left(r \mathbb{S}^{d-1}\right) .
$$

The complete description of the above equilibria of the average collision operator $Q$, called the von Mises-Fisher distributions, is given by Proposition 1.4 whose proof is detailed below. We start with the following easy integration by parts formula on spheres. The proof is postponed to Appendix A.

Lemma 2.1. Assume that $A=A(v)$ is a $C^{1}$-vector field in $\mathcal{O}=\left\{v \in \mathbb{R}^{d}: r_{1}<\right.$ $\left.|v|<r_{2}\right\}$. Then for any $\left.t \in\right] r_{1}, r_{2}[$ we have

$$
\int_{|\omega|=t}\left(\operatorname{div}_{v} A\right)(\omega) \mathrm{d} \omega=\int_{|\omega|=t}\left\{\frac{\omega \otimes \omega}{t^{2}}: \partial_{v} A(\omega)+\frac{(d-1) \omega}{t^{2}} \cdot A(\omega)\right\} \mathrm{d} \omega .
$$

In particular, if $A(v) \cdot v=0, v \in \mathcal{O}$, then

$$
\left.\int_{|\omega|=t}\left(\operatorname{div}_{v} A\right)(\omega) \mathrm{d} \omega=0, \quad t \in\right] r_{1}, r_{2}[
$$

and for any function $\chi \in C^{1}(\mathcal{O})$ we have

$$
\left.\int_{|\omega|=t} \nabla_{v} \chi(\omega) \cdot A(\omega) \mathrm{d} \omega+\int_{|\omega|=t} \chi(\omega)\left(\operatorname{div}_{v} A\right)(\omega) \mathrm{d} \omega=0, \quad t \in\right] r_{1}, r_{2}[.
$$

It is very convenient to express the differential operators $\nabla_{\omega}, \operatorname{div}_{\omega}$ of functions and vector fields on the sphere $r \mathbb{S}^{d-1}$ in terms of the differential operators $\nabla_{v}, \operatorname{div}_{v}$ applied to extensions of functions and vector fields on a neighborhood of $r \mathbb{S}^{d-1}$ in $\mathbb{R}^{d}$. The notation $\sim$ stands for the restriction on the sphere $r \mathbb{S}^{d-1}$ and ${ }^{\tau}$ for the restriction on the sphere $t \mathbb{S}^{d-1}$. The proof of the following lemma is detailed in Appendix B.

Lemma 2.2. (1) Let $\psi=\psi(v)$ be a $C^{1}$-function in an open set of $\mathbb{R}^{d}$, containing $r \mathbb{S}^{d-1}$. Then, for any $\omega \in r \mathbb{S}^{d-1}$ we have

$$
\nabla_{\omega} \widetilde{\psi}(\omega)=\left(I_{d}-\frac{\omega \otimes \omega}{r^{2}}\right) \widetilde{\nabla_{v} \psi}(\omega)
$$

(2) Let $\widetilde{\psi}=\widetilde{\psi}(\omega)$ be a $C^{1}$-function on $r \mathbb{S}^{d-1}$ and $\psi: \mathcal{O}=\left\{v \in \mathbb{R}^{d}: r_{1}<|v|<\right.$ $\left.r_{2}\right\} \rightarrow \mathbb{R}$ be the function defined by $\psi(v)=\widetilde{\psi}\left(r \frac{v}{|v|}\right), v \in \mathcal{O}$, with $0<r_{1}<r<r_{2}<$ $+\infty$. Then, for any $t \in] r_{1}, r_{2}[$, we have

$$
\left(\nabla_{v} \psi\right)\left(\omega_{t}\right)=\left(\nabla_{\omega_{t}} \widetilde{\psi}^{t}\right)\left(\omega_{t}\right)=\frac{r}{t}\left(\nabla_{\omega} \widetilde{\psi}\right)\left(r \frac{\omega_{t}}{t}\right), \quad\left|\omega_{t}\right|=t .
$$

(3) Let $\widetilde{\xi}=\widetilde{\xi}(\omega)$ be a $C^{1}$-tangent vector field on $r \mathbb{S}^{d-1}$ and $\xi=\xi(v)$ a $C^{1}$-extension of $\widetilde{\xi}$ in the set $\mathcal{O}=\left\{v \in \mathbb{R}^{d}: r_{1}<|v|<r_{2}\right\}$ such that $\xi(v) \cdot v=0$ for any $v \in \mathcal{O}$. Then we have

$$
\left(\operatorname{div}_{\omega} \widetilde{\xi}\right)(\omega)=\left(\widetilde{\operatorname{div}_{v} \xi}\right)(\omega), \quad \omega \in r \mathbb{S}^{d-1}
$$


(4) Let $\widetilde{\xi}=\widetilde{\xi}(\omega)$ be a $C^{1}$-tangent vector field on $r \mathbb{S}^{d-1}$ and $\xi(v)=\widetilde{\xi}\left(r \frac{v}{|v|}\right), v \in$ $\mathbb{R}^{d} \backslash\{0\}$, then

$$
\left(\operatorname{div}_{\omega_{t}} \xi\right)\left(\omega_{t}\right)=\frac{r}{t}\left(\operatorname{div}_{\omega} \widetilde{\xi}\right)\left(\frac{r}{t} \omega_{t}\right), \quad\left|\omega_{t}\right|=t .
$$

Before giving the proof of Proposition 1.4. we indicate a formula which will be used several times in our computations. For any continuous function $G:[-r, r] \rightarrow$ $\mathbb{R}, d \geq 2, \Omega \in \mathbb{S}^{d-1}$, we have

$$
\int_{r \mathbb{S}^{d-1}} G(\omega \cdot \Omega) \mathrm{d} \omega=\int_{0}^{\pi} G(r \cos \theta) \sin ^{d-2} \theta \mathrm{d} \theta r^{d-1} \bar{\omega}_{d-1}
$$

with $\omega_{1}=2$. In particular, for any continuous function $g:[-r, r] \rightarrow \mathbb{R}$, we have

$$
\begin{aligned}
\int_{r \mathbb{S}^{d-1}} g(\omega \cdot \Omega) M_{l \Omega}(\omega) \mathrm{d} \omega & =\frac{\int_{r \mathbb{S}^{d-1}} g(\omega \cdot \Omega) \exp \left(l \Omega \cdot \frac{\omega}{r}\right) \mathrm{d} \omega}{\int_{r \mathbb{S}^{d-1}} \exp \left(l \Omega \cdot \frac{\omega}{r}\right) \mathrm{d} \omega} \\
& =\frac{\int_{0}^{\pi} g(r \cos \theta) e^{l \cos \theta} \sin ^{d-2} \theta \mathrm{d} \theta}{\int_{0}^{\pi} e^{l \cos \theta} \sin ^{d-2} \theta \mathrm{d} \theta} .
\end{aligned}
$$

Proof of Proposition 1.4. (1) $\Rightarrow(2)$ We assume that $F$ is an equilibrium for the average collision kernel. We claim that $\int_{\mathbb{R}^{d}} \varphi(v) F \mathrm{~d} v=0$ for any continuous function $\varphi$ satisfying $\int_{r \mathbb{S}^{d-1}} \varphi(\omega) M(\omega) \mathrm{d} \omega=0$, with $M(v)=\exp \left(-\frac{|v-u[F]|^{2}}{2 \sigma}\right), v \in \mathbb{R}^{d}$. The idea is to solve the problem

$$
-\operatorname{div}_{\omega}\left(M(\omega) \nabla_{\omega} \widetilde{\psi}\right)=M(\omega) \widetilde{\varphi}(\omega), \quad \omega \in r \mathbb{S}^{d-1},
$$

where $\widetilde{\varphi}$ is the restriction on $r \mathbb{S}^{d-1}$ of $\varphi$ as usual. Notice that we have

$$
\int_{r \mathbb{S}^{d-1}} \tilde{\varphi}(\omega) M(\omega) \mathrm{d} \omega=\int_{r \mathbb{S}^{d-1}} \varphi(\omega) M(\omega) \mathrm{d} \omega=0
$$

We introduce the Hilbert spaces:

$$
\begin{aligned}
L^{2}\left(r \mathbb{S}^{d-1}\right) & =\left\{\chi: r \mathbb{S}^{d-1} \rightarrow \mathbb{R}, \int_{r \mathbb{S}^{d-1}} \chi^{2}(\omega) M(\omega) \mathrm{d} \omega<+\infty\right\}, \\
H^{1}\left(r \mathbb{S}^{d-1}\right) & =\left\{\chi: r \mathbb{S}^{d-1} \rightarrow \mathbb{R}, \int_{r \mathbb{S}^{d-1}}\left\{\chi^{2}+\left|\nabla_{\omega} \chi\right|^{2}\right\}(\omega) M(\omega) \mathrm{d} \omega<+\infty\right\},
\end{aligned}
$$

endowed with the scalar products:

$$
\begin{aligned}
(\chi, \theta)_{r} & =\int_{r \mathbb{S}^{d-1}} \chi(\omega) \theta(\omega) M(\omega) \mathrm{d} \omega, \quad \chi, \theta \in L^{2}\left(r \mathbb{S}^{d-1}\right), \\
((\chi, \theta))_{r} & =\int_{r \mathbb{S}^{d-1}}\left[\chi(\omega) \theta(\omega)+\nabla_{\omega} \chi \cdot \nabla_{\omega} \theta\right] M(\omega) \mathrm{d} \omega, \quad \chi, \theta \in H^{1}\left(r \mathbb{S}^{d-1}\right) .
\end{aligned}
$$

We denote by $|\cdot|_{r},\|\cdot\|_{r}$ the norm induced by the above scalar products. There is a constant $C_{r}$ such that the following Poincaré inequality holds true:

$$
|\chi|_{r}^{2}=\int_{r \mathbb{S}^{d-1}}(\chi(\omega))^{2} M(\omega) \mathrm{d} \omega \leq C_{r} \int_{r \mathbb{S}^{d-1}}\left|\nabla_{\omega} \chi\right|^{2} M(\omega) \mathrm{d} \omega=C_{r}\left|\nabla_{\omega} \chi\right|_{r}^{2},
$$


for any $\chi \in H^{1}\left(r \mathbb{S}^{d-1}\right)$ satisfying $\int_{r \mathbb{S}^{d-1}} \chi(\omega) M(\omega) \mathrm{d} \omega=0$. The previous inequality guarantees that the application $\chi \rightarrow\left|\nabla_{\omega} \chi\right|_{r}$ is a norm equivalent to $\|\cdot\|_{r}$ on

$$
\tilde{H}^{1}\left(r \mathbb{S}^{d-1}\right):=H^{1}\left(r \mathbb{S}^{d-1}\right) \cap\left\{\theta \in L^{2}\left(r \mathbb{S}^{d-1}\right): \int_{r \mathbb{S}^{d-1}} \theta(\omega) M(\omega) \mathrm{d} \omega=0\right\} .
$$

Therefore, the bilinear form

$$
(\chi, \theta) \in \tilde{H}^{1}\left(r \mathbb{S}^{d-1}\right) \times \tilde{H}^{1}\left(r \mathbb{S}^{d-1}\right) \rightarrow \int_{r \mathbb{S}^{d-1}} \nabla_{\omega} \chi \cdot \nabla_{\omega} \theta M(\omega) \mathrm{d} \omega
$$

is symmetric, bounded and coercive. By the Lax-Milgram lemma, there is a unique solution $\widetilde{\psi} \in \tilde{H}^{1}\left(r \mathbb{S}^{d-1}\right)$ for the variational problem (2.6) leading to

$$
\int_{r \mathbb{S}^{d-1}} \nabla_{\omega} \widetilde{\psi} \cdot \nabla_{\omega} \chi M(\omega) \mathrm{d} \omega=\int_{r \mathbb{S}^{d-1}} \widetilde{\varphi}(\omega) \chi(\omega) M(\omega) \mathrm{d} \omega
$$

for any $\chi \in \tilde{H}^{1}\left(r \mathbb{S}^{d-1}\right)$. Observe that (2.7) still holds true for any constant function on $r \mathbb{S}^{d-1}$, thanks to the compatibility condition $\int_{r \mathbb{S}^{d-1}} \widetilde{\varphi}(\omega) M(\omega) \mathrm{d} \omega=0$. Therefore the variational formulation is valid for any function $\chi \in H^{1}\left(r \mathbb{S}^{d-1}\right)$, implying that

$$
-\operatorname{div}_{\omega}\left(M(\omega) \nabla_{\omega} \tilde{\psi}\right)=M(\omega) \widetilde{\varphi}(\omega), \quad \omega \in r \mathbb{S}^{d-1} .
$$

We consider the extension of $\widetilde{\psi}$ defined as usual as

$$
\psi(v)=\widetilde{\psi}\left(r \frac{v}{|v|}\right), \quad v \in \mathbb{R}^{d} \backslash\{0\} .
$$

By Lemma 2.2 statements (2) and (3), we check that for any $v \in r \mathbb{S}^{d-1}$ we have

$$
\begin{aligned}
M(v)\left\{\frac{v-u[F]}{\sigma} \cdot \nabla_{v}\left[\widetilde{\psi}\left(r \frac{v}{|v|}\right)\right]-\Delta_{v}\left[\widetilde{\psi}\left(r \frac{v}{|v|}\right)\right]\right\} & =-\operatorname{div}_{\omega}\left(M \nabla_{\omega} \widetilde{\psi}\right) \\
& =M(v) \widetilde{\varphi}(v)
\end{aligned}
$$

and therefore we obtain

$$
\int_{\mathbb{R}^{d}} \varphi(v) F \mathrm{~d} v=\int_{\mathbb{R}^{d}}\left\{\frac{v-u[F]}{\sigma} \cdot \nabla_{v}\left[\widetilde{\psi}\left(r \frac{v}{|v|}\right)\right]-\Delta_{v}\left[\widetilde{\psi}\left(r \frac{v}{|v|}\right)\right]\right\} F \mathrm{~d} v=0 .
$$

We deduce that the linear forms $\varphi \rightarrow \int_{r \mathbb{S}^{d-1}} \varphi(\omega) M(\omega) \mathrm{d} \omega$ and $\varphi \rightarrow \int_{\mathbb{R}^{d}} \varphi(v) F \mathrm{~d} v$ are proportional, see Lemma III.2 in Ref. 22 , and thus there is $\tilde{C}$ such that for any $\varphi \in C\left(\mathbb{R}^{d}\right)$, we have

$$
\int_{\mathbb{R}^{d}} \varphi(v) F \mathrm{~d} v=\tilde{C} \int_{r \mathbb{S}^{d-1}} \varphi(\omega) M(\omega) \mathrm{d} \omega=\rho \frac{\int_{r \mathbb{S}^{d-1}} \varphi(\omega) \exp \left(\frac{\omega \cdot u[F]}{\sigma}\right) \mathrm{d} \omega}{\int_{r \mathbb{S}^{d-1}} \exp \left(\frac{\omega \cdot u[F]}{\sigma}\right) \mathrm{d} \omega}
$$

with $\rho=\tilde{C} \int_{r \mathbb{S}^{d-1}} M(\omega) \mathrm{d} \omega$. Therefore the measure $F$ has a positive density with respect to $\mathrm{d} \omega$ on $r \mathbb{S}^{d-1}$ :

$$
F=\rho \frac{\exp \left(\frac{\omega \cdot u[F]}{\sigma}\right) \mathrm{d} \omega}{\int_{r \mathbb{S}^{d-1}} \exp \left(\frac{\omega^{\prime} \cdot u[F]}{\sigma}\right) \mathrm{d} \omega^{\prime}} .
$$

If $\rho=0$, we obtain $F=0$, and we can take $l=0$ and any $\Omega \in \mathbb{S}^{d-1}$. Assume now that $\rho>0$. If $u[F]=0$, we obtain $F=\rho \frac{\mathrm{d} \omega}{\omega_{d} r^{d-1}}$ which corresponds to $l=0$ 
and any $\Omega \in \mathbb{S}^{d-1}$. If $u[F] \neq 0$, we introduce $\Omega[F]=\frac{u[F]}{|u[F]|}$. By the definition of $u[F]$, we have

$$
\begin{aligned}
u[F] & =\frac{\int_{r \mathbb{S}^{d-1}} \exp \left(\frac{\omega \cdot u[F]}{\sigma}\right) \omega \mathrm{d} \omega}{\int_{r \mathbb{S}^{d-1}} \exp \left(\frac{\omega \cdot u[F]}{\sigma}\right) \mathrm{d} \omega} \\
& =\frac{\int_{0}^{\pi} r \cos \theta \exp \left(\frac{r|u[F]|}{\sigma} \cos \theta\right) \sin ^{d-2} \theta \mathrm{d} \theta}{\int_{0}^{\pi} \exp \left(\frac{r|u[F]|}{\sigma} \cos \theta\right) \sin ^{d-2} \theta \mathrm{d} \theta} \Omega[F] .
\end{aligned}
$$

For the last equality use the fact that

$$
\int_{r \mathbb{S}^{d-1}} \exp \left(\frac{\omega \cdot u[F]}{\sigma}\right) \omega \mathrm{d} \omega=\int_{r \mathbb{S}^{d-1}} \exp \left(\frac{\omega \cdot u[F]}{\sigma}\right)(\omega \cdot \Omega) \mathrm{d} \omega \Omega
$$

and formula (2.5). The equality (2.8) reduces to the condition

$$
\frac{|u[F]|}{r}=\frac{\int_{0}^{\pi} \cos \theta \exp \left(\frac{r|u[F]|}{\sigma} \cos \theta\right) \sin ^{d-2} \theta \mathrm{d} \theta}{\int_{0}^{\pi} \exp \left(\frac{r|u[F]|}{\sigma} \cos \theta\right) \sin ^{d-2} \theta \mathrm{d} \theta} .
$$

We introduce the function $\lambda: \mathbb{R}_{+} \rightarrow \mathbb{R}:$

$$
\lambda(l)=\frac{\int_{0}^{\pi} \cos \theta e^{l \cos \theta} \sin ^{d-2} \theta \mathrm{d} \theta}{\int_{0}^{\pi} e^{l \cos \theta} \sin ^{d-2} \theta \mathrm{d} \theta}, \quad l \in \mathbb{R}_{+} .
$$

Therefore the non-negative number $l=\frac{r|u[F]|}{\sigma}$ satisfies $\lambda(l)=\frac{\sigma}{r^{2}} l$, and thus the measure $F$ is given by

$$
F=\rho \frac{\exp \left(\frac{r|u[F]|}{\sigma} \frac{\omega}{r} \cdot \Omega\right) \mathrm{d} \omega}{\int_{r \mathbb{S}^{d-1}} \exp \left(\frac{r|u[F]|}{\sigma} \frac{\omega^{\prime}}{r} \cdot \Omega\right) \mathrm{d} \omega^{\prime}}=\rho M_{l \Omega} \mathrm{d} \omega
$$

with $\rho \in \mathbb{R}_{+}, \Omega=\frac{u[F]}{|u[F]|} \in \mathbb{S}^{d-1}, l \in \mathbb{R}_{+}$satisfying $\lambda(l)=\frac{\sigma}{r^{2}} l$.

$(2) \Rightarrow(1)$ Conversely, let $F$ be a measure given by $F=\rho M_{l \Omega} \mathrm{d} \omega$ for some $\rho \in \mathbb{R}_{+}$, $\Omega \in \mathbb{S}^{d-1}, l \in \mathbb{R}_{+}$such that $\lambda(l)=\frac{\sigma}{r^{2}} l$. If $\rho=0, F$ is the trivial equilibrium (with $u[F]=0)$. If $\rho>0$, the mean velocity writes

$$
\begin{aligned}
u[F] & =\frac{\int_{\mathbb{R}^{d}} v F \mathrm{~d} v}{\int_{\mathbb{R}^{d}} F \mathrm{~d} v}=\frac{\int_{r \mathbb{S}^{d-1}}(\omega \cdot \Omega) \exp \left(l \frac{\omega}{r} \cdot \Omega\right) \mathrm{d} \omega}{\int_{r \mathbb{S}^{d-1}} \exp \left(l \frac{\omega}{r} \cdot \Omega\right) \mathrm{d} \omega} \Omega \\
& =\frac{r \int_{0}^{\pi} \cos \theta e^{l \cos \theta} \sin ^{d-2} \theta \mathrm{d} \theta}{\int_{0}^{\pi} e^{l \cos \theta} \sin ^{d-2} \theta \mathrm{d} \theta} \Omega=r \lambda(l) \Omega=\frac{\sigma}{r} l \Omega,
\end{aligned}
$$

saying that $\frac{u[F]}{|u[F]|}=\Omega$ and $|u[F]|=\frac{\sigma l}{r}$. For any test function $\widetilde{\psi} \in C^{2}\left(r \mathbb{S}^{d-1}\right)$ we have

$$
\begin{aligned}
M(v) & {\left[(v-u[F]) \cdot \nabla_{v}\left[\widetilde{\psi}\left(r \frac{v}{|v|}\right)\right]-\sigma \Delta_{v}\left[\widetilde{\psi}\left(r \frac{v}{|v|}\right)\right]\right] } \\
= & -\sigma \operatorname{div}_{\omega}\left(M \nabla_{\omega} \widetilde{\psi}\right), \quad v \in r \mathbb{S}^{d-1}
\end{aligned}
$$


where $M(v)=\exp \left(-\frac{|v-u[F]|^{2}}{2 \sigma}\right), v \in \mathbb{R}^{d}$. Notice that for any $v \in r \mathbb{S}^{d-1}$ we have

$$
M(v)=\exp \left(-\frac{r^{2}+\frac{\sigma^{2} l^{2}}{r^{2}}}{2 \sigma}\right) \int_{r \mathbb{S}^{d-1}} \exp \left(l \Omega \cdot \frac{\omega}{r}\right) \mathrm{d} \omega M_{l \Omega}(\omega)
$$

and thus, the above equality becomes

$$
M_{l \Omega}(v)\left\{(v-u[F]) \cdot \nabla_{v}\left[\widetilde{\psi}\left(r \frac{v}{|v|}\right)\right]-\sigma \Delta_{v}\left[\widetilde{\psi}\left(r \frac{v}{|v|}\right)\right]\right\}=-\sigma \operatorname{div}_{\omega}\left(M_{l \Omega} \nabla_{\omega} \widetilde{\psi}\right) .
$$

Therefore we obtain

$$
\begin{aligned}
\int_{v \neq 0} & \left\{(v-u[F]) \cdot \nabla_{v}\left[\widetilde{\psi}\left(r \frac{v}{|v|}\right)\right]-\sigma \Delta_{v}\left[\widetilde{\psi}\left(r \frac{v}{|v|}\right)\right]\right\} F \mathrm{~d} v \\
= & \int_{|v|=r}\left\{(v-u[F]) \cdot \nabla_{v}\left[\widetilde{\psi}\left(r \frac{v}{|v|}\right)\right]-\sigma \Delta_{v}\left[\widetilde{\psi}\left(r \frac{v}{|v|}\right)\right]\right\} \rho M_{l \Omega}(v) \mathrm{d} v \\
= & -\rho \sigma \int_{r \mathbb{S}^{d-1}} \operatorname{div}_{\omega}\left(M_{l \Omega}(\omega) \nabla_{\omega} \widetilde{\psi}\right) \mathrm{d} \omega=0 .
\end{aligned}
$$

The properties of the function $\lambda$ are summarized in Proposition 1.5, whose proof is detailed below.

Proof of Proposition 1.5. We introduce the function

$$
\beta_{0}(l)=\frac{1}{\pi} \int_{0}^{\pi} e^{l \cos \theta} \sin ^{d-2} \theta \mathrm{d} \theta, \quad l \in \mathbb{R} .
$$

It is a Bessel-like function. ${ }^{1}$ Indeed, it verifies the linear second-order differential equation

$$
l^{2} \beta_{0}^{\prime \prime}(l)+(d-1) l \beta_{0}^{\prime}(l)=l^{2} \beta_{0}(l), \quad l \in \mathbb{R} .
$$

We recall that the standard modified Bessel function $I_{n}(l)=\frac{1}{\pi} \int_{0}^{\pi} e^{l \cos \theta} \cos (n \theta) \mathrm{d} \theta$, $n \in \mathbb{N}$, satisfy

$$
l^{2} I_{n}^{\prime \prime}(l)+l I_{n}^{\prime}(l)=\left(l^{2}+n^{2}\right) I_{n}(l), \quad l \in \mathbb{R} .
$$

Clearly $\beta_{0}^{\prime}(l)=\frac{1}{\pi} \int_{0}^{\pi} \cos \theta e^{l \cos \theta} \sin ^{d-2} \theta \mathrm{d} \theta$ and thus the function $\lambda$ writes

$$
\lambda(l)=\frac{\beta_{0}^{\prime}(l)}{\beta_{0}(l)} .
$$

It is easily seen that $\beta_{0}^{\prime}(0)=0$, implying that $\lambda(0)=0$. Indeed, we have

$$
\pi \beta_{0}^{\prime}(0)=\int_{0}^{\pi} \cos \theta \sin ^{d-2} \theta \mathrm{d} \theta=\int_{0}^{\pi} \frac{\mathrm{d}}{\mathrm{d} \theta} \frac{\sin ^{d-1} \theta}{d-1} \mathrm{~d} \theta=0, \quad d \geq 2 .
$$

Moreover, $\lambda$ is strictly increasing. This comes by the formula

$$
\lambda^{\prime}(l)=\frac{\beta_{0}^{\prime \prime}(l) \beta_{0}(l)-\left(\beta_{0}^{\prime}(l)\right)^{2}}{\beta_{0}^{2}(l)}
$$


and by observing that the Cauchy inequality implies

$$
\begin{aligned}
\left(\beta_{0}^{\prime}(l)\right)^{2} & =\left(\frac{1}{\pi} \int_{0}^{\pi} \cos \theta e^{l \cos \theta} \sin ^{d-2} \theta \mathrm{d} \theta\right)^{2} \\
& <\frac{1}{\pi} \int_{0}^{\pi} e^{l \cos \theta} \sin ^{d-2} \theta \mathrm{d} \theta \frac{1}{\pi} \int_{0}^{\pi} \cos ^{2} \theta e^{l \cos \theta} \sin ^{d-2} \theta \mathrm{d} \theta=\beta_{0}(l) \beta_{0}^{\prime \prime}(l) .
\end{aligned}
$$

The derivative of $\lambda$ at $l=0$ is

$$
\begin{aligned}
\lambda^{\prime}(0) & =\frac{\beta_{0}^{\prime \prime}(0)}{\beta_{0}(0)}=\frac{\int_{0}^{\pi} \cos ^{2} \theta \sin ^{d-2} \theta \mathrm{d} \theta}{\int_{0}^{\pi} \sin ^{d-2} \theta \mathrm{d} \theta}=\frac{\int_{0}^{\pi} \cos \theta \frac{\mathrm{d}}{\mathrm{d} \theta} \frac{\sin ^{d-1} \theta}{d-1} \mathrm{~d} \theta}{\int_{0}^{\pi} \sin ^{d-2} \theta \mathrm{d} \theta} \\
& =\frac{\int_{0}^{\pi} \sin ^{d} \theta \mathrm{d} \theta}{(d-1) \int_{0}^{\pi} \sin ^{d-2} \theta \mathrm{d} \theta} .
\end{aligned}
$$

Using $\sin ^{2} \theta+\cos ^{2} \theta=1$ in the first equality above, we also have

$$
\lambda^{\prime}(0)=1-\frac{\int_{0}^{\pi} \sin ^{d} \theta \mathrm{d} \theta}{\int_{0}^{\pi} \sin ^{d-2} \theta \mathrm{d} \theta} .
$$

We deduce that

$$
\frac{\int_{0}^{\pi} \sin ^{d} \theta \mathrm{d} \theta}{\int_{0}^{\pi} \sin ^{d-2} \theta \mathrm{d} \theta}=1-\lambda^{\prime}(0)=(d-1) \lambda^{\prime}(0),
$$

which yields $\lambda^{\prime}(0)=1 / d$. We claim that the function $\zeta: \mathbb{R}_{+} \rightarrow \mathbb{R}$, defined by $\zeta(l)=\frac{\lambda(l)}{l}, l>0$ and $\zeta(0)=\lim _{l \searrow 0} \frac{\lambda(l)}{l}=\lambda^{\prime}(0)=\frac{1}{d}$, is strictly decreasing on $\mathbb{R}_{+}$. Combining 2.10), (2.9), we obtain for any $l>0$ :

$$
\lambda^{\prime}(l)=\frac{\left(\beta_{0}(l)-\frac{d-1}{l} \beta_{0}^{\prime}(l)\right) \beta_{0}(l)}{\beta_{0}^{2}(l)}-\left(\frac{\beta_{0}^{\prime}(l)}{\beta_{0}(l)}\right)^{2}=1-\frac{d-1}{l} \lambda(l)-\lambda^{2}(l),
$$

which implies after replacing $\lambda(l)$ by $l \zeta(l)$ :

$$
l \zeta^{\prime}(l)=1-d \zeta(l)-l^{2} \zeta^{2}(l), \quad l>0 .
$$

We are done if we prove that

$$
l^{2} \zeta^{2}(l)+d \zeta(l)-1>0, \quad l>0 .
$$

It is enough to check that

$$
\zeta(l)>\frac{\sqrt{d^{2}+4 l^{2}}-d}{2 l^{2}}, \quad l>0
$$

or equivalently

$$
\lambda(l)>\frac{\sqrt{d^{2}+4 l^{2}}-d}{2 l}, \quad l>0,
$$

which is one of the statements of Lemma 2.3 Clearly the function $\lambda$ is bounded on $\mathbb{R}_{+}$:

$$
0=\lambda(0)<\lambda(l)=\frac{\int_{0}^{\pi} \cos \theta e^{l \cos \theta} \sin ^{d-2} \theta \mathrm{d} \theta}{\int_{0}^{\pi} e^{l \cos \theta} \sin ^{d-2} \theta \mathrm{d} \theta}<1
$$


By 2.11) we deduce that the function $l \rightarrow \lambda^{\prime}(l)$ has a limit when $l \rightarrow+\infty$, and

$$
\lim _{l \rightarrow+\infty} \lambda^{\prime}(l)=1-\lim _{l \rightarrow+\infty} \lambda^{2}(l) .
$$

Let us denote by $\Lambda_{0}, \Lambda_{1}$ the limits

$$
\left.\left.\Lambda_{0}=\lim _{l \rightarrow+\infty} \lambda(l) \in\right] 0,1\right], \quad \Lambda_{1}=\lim _{l \rightarrow+\infty} \lambda^{\prime}(l) .
$$

We know that $\Lambda_{1}=1-\Lambda_{0}^{2}$. Therefore we obtain $\Lambda_{0}=1$, provided that $\Lambda_{1}=0$. For any $l>0$, there is $\left.y_{l} \in\right] 0, l\left[\right.$ such that $\frac{\lambda(l)}{l}=\lambda^{\prime}\left(y_{l}\right)$. We claim that $\lim _{l \rightarrow+\infty} y_{l}=$ $+\infty$. Indeed, for any $Y>0$, there is $L>0$ such that

$$
\inf _{y \in[0, Y]} \lambda^{\prime}(y)>\frac{1}{L}
$$

Let $l>L$. Assuming that $y_{l} \in[0, Y]$ leads to a contradiction, since

$$
\frac{1}{l}>\frac{\lambda(l)}{l}=\lambda^{\prime}\left(y_{l}\right) \geq \inf _{y \in[0, Y]} \lambda^{\prime}(y)>\frac{1}{L}>\frac{1}{l} .
$$

Therefore, for any $Y>0$, there is $L>0$ such that $l>L$ implies $y_{l}>Y$, saying that $\lim _{l \rightarrow+\infty} y_{l}=+\infty$. As we already know that $\lambda^{\prime}$ has a limit when $l \rightarrow+\infty$, and by the boundedness of $\lambda$, we deduce that

$$
\Lambda_{1}=\lim _{l \rightarrow+\infty} \lambda^{\prime}\left(y_{l}\right)=\lim _{l \rightarrow+\infty} \frac{\lambda(l)}{l}=0 .
$$

If $\frac{\sigma}{r^{2}} \geq \frac{1}{d}$, the strict monotonicity of $\zeta$ implies

$$
\frac{\lambda(l)}{l}=\zeta(l)<\zeta(0)=\lambda^{\prime}(0)=\frac{1}{d} \leq \frac{\sigma}{r^{2}}, \quad l>0,
$$

saying that the only solution of $\lambda(l)=\frac{\sigma}{r^{2}} l$ on $\mathbb{R}_{+}$is $l=0$. If $\left.\frac{\sigma}{r^{2}} \in\right] 0, \frac{1}{d}[$ we have

$$
\zeta(0)=\frac{1}{d}>\frac{\sigma}{r^{2}}>0=\lim _{l \rightarrow+\infty} \zeta(l)
$$

and the continuity and strict monotonicity of $\zeta$ imply that there is a unique solution $l\left(\frac{\sigma}{r^{2}}\right)>0$ such that $\lambda\left(l\left(\frac{\sigma}{r^{2}}\right)\right)=\frac{\sigma}{r^{2}} l\left(\frac{\sigma}{r^{2}}\right)$.

Remark 2.1. The value $l=0$ corresponds to the isotropic equilibrium $M_{0 \Omega} \mathrm{d} \omega=$ $\frac{\mathrm{d} \omega}{\bar{\omega}_{d} r^{d-1}}$. The limit when $l \rightarrow+\infty$ leads to the Dirac measure on $r \mathbb{S}^{d-1}$, concentrated at $r \Omega$, that is, for any function $\widetilde{\psi} \in C\left(r \mathbb{S}^{d-1}\right)$ we have

$$
\lim _{l \rightarrow+\infty} \int_{r \mathbb{S}^{d-1}} \widetilde{\psi}(\omega) M_{l \Omega}(\omega) \mathrm{d} \omega=\widetilde{\psi}(r \Omega) .
$$

The function $\lambda$ can be computed explicitly, at least for $d=3$. Nevertheless, very good explicit approximations are available in any dimension $d$. 
Lemma 2.3. (1) Consider the function

$$
\mu: \mathbb{R}_{+} \rightarrow \mathbb{R}_{+}, \quad \mu(l)=\frac{\sqrt{d^{2}+4 l^{2}}-d}{2 l}=\frac{2 l}{\sqrt{d^{2}+4 l^{2}}+d}, \quad l \in \mathbb{R}_{+} .
$$

The function $\mu$ is strictly increasing, strictly concave and we have:

$$
\begin{aligned}
& \mu(0)=\lambda(0)=0, \quad \mu^{\prime}(0)=\lambda^{\prime}(0)=\frac{1}{d}, \quad \lim _{l \rightarrow+\infty} \mu(l)=1, \\
& \mu^{\prime}(l)<1-\frac{d-1}{l} \mu(l)-\mu^{2}(l), \quad \mu(l)<\lambda(l), \quad l>0 .
\end{aligned}
$$

(2) If $d=3$, the function $\lambda$ is given by $\lambda(l)=\frac{\cosh (l)}{\sinh (l)}-\frac{1}{l}, l>0$.

Proof. (1) By direct computations we obtain

$$
\mu^{\prime}(l)=\frac{2 d}{\sqrt{d^{2}+4 l^{2}}\left(\sqrt{d^{2}+4 l^{2}}+d\right)}>0, \quad l \in \mathbb{R}_{+}
$$

and

$$
1-\frac{d-1}{l} \mu(l)-\mu^{2}(l)=\frac{2}{\sqrt{d^{2}+4 l^{2}}+d} .
$$

Therefore $\mu$ satisfies the first-order differential inequation

$$
\begin{aligned}
\mu^{\prime}(l) & =\frac{2 d}{\sqrt{d^{2}+4 l^{2}}\left(\sqrt{d^{2}+4 l^{2}}+d\right)}<\frac{2}{\sqrt{d^{2}+4 l^{2}}+d} \\
& =1-\frac{d-1}{l} \mu(l)-\mu^{2}(l), \quad l>0
\end{aligned}
$$

and the initial condition $\mu(0)=0$. Recall that $\lambda$ satisfies the first-order differential equation (cf. (2.11):

$$
\lambda^{\prime}(l)=1-\frac{d-1}{l} \lambda(l)-\lambda^{2}(l), \quad l>0,
$$

with the initial condition $\lambda(0)=0$. By comparison principle, it follows that $\mu(l)<$ $\lambda(l)$ for any $l>0$. Clearly $\mu^{\prime}(0)=\frac{1}{d}=\lambda^{\prime}(0), \lim _{l \rightarrow+\infty} \mu(l)=1, \mu^{\prime}(l)>0, l \in \mathbb{R}_{+}$, and $\mu^{\prime}$ is strictly decreasing, saying that $\mu$ is strictly increasing and strictly concave on $\mathbb{R}_{+}$.

(2) In the case $d=3$ we obtain:

$$
\begin{aligned}
& \pi \beta_{0}(l)=\int_{0}^{\pi} e^{l \cos \theta} \sin \theta \mathrm{d} \theta=\frac{e^{l}-e^{-l}}{l}, \quad l>0, \\
& \pi \beta_{0}^{\prime}(l)=\int_{0}^{\pi} e^{l \cos \theta} \cos \theta \sin \theta \mathrm{d} \theta=\frac{e^{l}+e^{-l}}{l}-\frac{e^{l}-e^{-l}}{l^{2}}, \quad l>0,
\end{aligned}
$$

implying that

$$
\lambda(l)=\frac{\beta_{0}^{\prime}(l)}{\beta_{0}(l)}=\frac{\cosh (l)}{\sinh (l)}-\frac{1}{l}, \quad l>0 .
$$


In order to exploit the constraint (1.8) we will need to compute $Q(F)$, where $F$ is a von Mises-Fisher equilibrium, let us say $F=M_{l \Omega}(\omega) \mathrm{d} \omega$. This computation is detailed in the following lemma. The notation $(\cdot, \cdot)$ stands for the pairing between distributions and smooth functions.

Lemma 2.4. Let $F=M_{l \Omega}(\omega) \mathrm{d} \omega$ be a von Mises-Fisher equilibrium. Then we have, for any function $\varphi \in C_{c}^{2}\left(\mathbb{R}^{d}\right)$ :

$$
(Q(F), \varphi)=\left.\sigma \frac{M_{l \Omega}}{M} \frac{\mathrm{d}}{\mathrm{d} t}\right|_{t=r} \int_{\left|\omega_{t}\right|=t} M\left(\omega_{t}\right)\left(\nabla_{v} \varphi\right)\left(\omega_{t}\right) \cdot \frac{\omega_{t}}{t} \mathrm{~d} \omega_{t},
$$

where $M(v)=\exp \left(-\frac{|v-u[F]|^{2}}{2 \sigma}\right), v \in \mathbb{R}^{d}$.

Proof. Pick a test function $\varphi \in C_{c}^{2}\left(\mathbb{R}^{d}\right)$ and notice that

$$
\begin{aligned}
(Q(F), \varphi) & =\left(F, \sigma \Delta_{v} \varphi-(v-u[F]) \cdot \nabla_{v} \varphi\right) \\
& =\left(F, \sigma \frac{\operatorname{div}_{v}\left(M \nabla_{v} \varphi\right)}{M(v)}\right) \\
& =\sigma \int_{r \mathbb{S}^{d-1}} \operatorname{div}_{v}\left(M \nabla_{v} \varphi\right)(\omega) \frac{M_{l \Omega}(\omega)}{M(\omega)} \mathrm{d} \omega .
\end{aligned}
$$

It is easily seen that the function $\frac{M_{l \Omega}}{M}$ is constant on the sphere $r \mathbb{S}^{d-1}$ :

$$
\frac{M_{l \Omega}(\omega)}{M(\omega)}=\frac{\exp \left(\frac{r^{2}+|u[F]|^{2}}{2 \sigma}\right)}{\int_{r \mathbb{S}^{d-1}} \exp \left(l \Omega \cdot \frac{\omega^{\prime}}{r}\right) \mathrm{d} \omega^{\prime}}, \quad \omega \in r \mathbb{S}^{d-1}
$$

and therefore we have

$$
\begin{aligned}
(Q(F), \varphi) & =\left.\sigma \frac{M_{l \Omega}}{M} \frac{\mathrm{d}}{\mathrm{d} t}\right|_{t=r} \int_{|v|<t} \operatorname{div}_{v}\left(M \nabla_{v} \varphi\right) \mathrm{d} v \\
& =\left.\sigma \frac{M_{l \Omega}}{M} \frac{\mathrm{d}}{\mathrm{d} t}\right|_{t=r} \int_{\left|\omega_{t}\right|=t} M\left(\omega_{t}\right) \nabla_{v} \varphi\left(\omega_{t}\right) \cdot \frac{\omega_{t}}{t} \mathrm{~d} \omega_{t} .
\end{aligned}
$$

Thanks to the above result, we can determine $F^{(1)}-\left\langle F^{(1)}\right\rangle$ in terms of $F$. More exactly we prove the following.

Lemma 2.5. Let $F=M_{l \Omega}(\omega) \mathrm{d} \omega$ be a von Mises-Fisher equilibrium and $F^{(1)}$ a bounded measure such that

$$
\operatorname{div}_{v}\left\{F^{(1)}\left(\alpha-\beta|v|^{2}\right) v\right\}=Q(F) .
$$

Then for any function $\chi \in C_{c}^{1}\left(\mathbb{R}^{d}\right)$, such that $\left.\chi\right|_{r \mathbb{S}^{d-1}}=0$ we have

$$
\begin{aligned}
\int_{\mathbb{R}^{d}} \chi(v)\left(F^{(1)}-\left\langle F^{(1)}\right\rangle\right) \mathrm{d} v & =\int_{v \neq 0} \chi(v) F^{(1)} \mathrm{d} v \\
& =\left.\sigma \frac{M_{l \Omega}}{M} \frac{\mathrm{d}}{\mathrm{d} t}\right|_{t=r} \int_{\left|\omega_{t}\right|=t} \frac{M\left(\omega_{t}\right) \chi\left(\omega_{t}\right)}{t \beta\left(t^{2}-r^{2}\right)} \mathrm{d} \omega_{t} .
\end{aligned}
$$


Proof. For any function $\varphi \in C_{c}^{1}\left(\mathbb{R}^{d}\right)$, we know that

$$
\begin{aligned}
-\int_{\mathbb{R}^{d}}\left(\alpha-\beta|v|^{2}\right) v \cdot \nabla_{v} \varphi F^{(1)} \mathrm{d} v & =(Q(F), \varphi) \\
& =\left.\sigma \frac{M_{l \Omega}}{M} \frac{\mathrm{d}}{\mathrm{d} t}\right|_{t=r} \int_{\left|\omega_{t}\right|=t} M\left(\omega_{t}\right) \nabla_{v} \varphi\left(\omega_{t}\right) \cdot \frac{\omega_{t}}{t} \mathrm{~d} \omega_{t} .
\end{aligned}
$$

The idea is to solve the adjoint problem (cf. Lemma 1.1):

$$
-\left(\alpha-\beta|v|^{2}\right) v \cdot \nabla_{v} \varphi=\chi(v)
$$

and to express the normal derivative of $\varphi$ in terms of $\chi$. Indeed, for any $\omega_{t} \in t \mathbb{S}^{d-1}$, we have

$$
\nabla_{v} \varphi\left(\omega_{t}\right) \cdot \frac{\omega_{t}}{t}=\frac{\chi\left(\omega_{t}\right)}{t\left(\beta t^{2}-\alpha\right)}=\frac{\chi\left(\omega_{t}\right)}{t \beta\left(t^{2}-r^{2}\right)}
$$

Finally we obtain the formula

$$
\int_{v \neq 0} \chi(v) F^{(1)} \mathrm{d} v=(Q(F), \varphi)=\left.\sigma \frac{M_{l \Omega}}{M} \frac{\mathrm{d}}{\mathrm{d} t}\right|_{t=r} \int_{\left|\omega_{t}\right|=t} \frac{M\left(\omega_{t}\right) \chi\left(\omega_{t}\right)}{t \beta\left(t^{2}-r^{2}\right)} \mathrm{d} \omega_{t} .
$$

Once we have determined the form of the dominant distribution $f(t, x, v)=$ $\rho(t, x) M_{l \Omega(t, x)} \mathrm{d} \omega$, we search for macroscopic equations characterizing $\rho(t, x)$ and $\Omega(t, x)$. For doing that, we use the moments of (1.10) with respect to the velocity. The key point is how to eliminate $f^{(1)}$ in the right-hand side of (1.10). Notice that this right-hand side is the linearization around $f$, with $\int_{\mathbb{R}^{d}} f \mathrm{~d} v>0$, computed in the direction $f^{(1)}$, of the average collision kernel $Q$ :

$$
\begin{aligned}
\mathcal{L}_{f}\left(f^{(1)}\right):=\lim _{\varepsilon \searrow 0} \frac{\left\langle Q\left(f+\varepsilon f^{(1)}\right)\right\rangle-\langle Q(f)\rangle}{\varepsilon}= & \left\langle\operatorname{div}_{v}\left[f^{(1)}(v-u[f])+\sigma \nabla_{v} f^{(1)}\right]\right\rangle \\
& -\left\langle\operatorname{div}_{v}\left[f \frac{\int_{\mathbb{R}^{d}} f^{(1)}\left(v^{\prime}-u[f]\right) \mathrm{d} v^{\prime}}{\int_{\mathbb{R}^{d}} f \mathrm{~d} v^{\prime}}\right]\right\rangle \\
& =\left\langle\operatorname{div}_{v} A_{f}\left(f^{(1)}\right)\right\rangle,
\end{aligned}
$$

where

$$
A_{f}\left(f^{(1)}\right)=\left[f^{(1)}(v-u[f])+\sigma \nabla_{v} f^{(1)}\right]-f \frac{\int_{\mathbb{R}^{d}} f^{(1)}\left(v^{\prime}-u[f]\right) \mathrm{d} v^{\prime}}{\int_{\mathbb{R}^{d}} f \mathrm{~d} v^{\prime}} .
$$

We are looking for functions such that

$$
\int_{\mathbb{R}^{d}} \psi(v)\left\langle\operatorname{div}_{v} A_{f}\left(f^{(1)}\right)\right\rangle \mathrm{d} v
$$

can be expressed in terms of the velocity moments of $f$, in order to get a closure for the macroscopic quantities $\rho(t, x), \Omega(t, x)$. For example $\psi(v)=1$ leads to the 
continuity equation

$$
\partial_{t} \int_{\mathbb{R}^{d}} f \mathrm{~d} v+\operatorname{div}_{x} \int_{\mathbb{R}^{d}} v f \mathrm{~d} v=0
$$

which also writes

$$
\partial_{t} \rho+\operatorname{div}_{x}\left(\rho \frac{\sigma}{r} l \Omega\right)=0 .
$$

Naturally, we need to find other functions $\psi$, which will allow us to characterize the time evolution of the orientation $\Omega$. Recall that the constraint (1.8) determines $f^{(1)}-\left\langle f^{(1)}\right\rangle$ (in terms of $f$ ), but not $\left\langle f^{(1)}\right\rangle$, as Lemma 2.5 implies. Motivated by this, we are looking for functions $\psi$ such that

$$
\int_{\mathbb{R}^{d}} \psi(v)\left\langle\operatorname{div}_{v} A_{f}\left(g^{(1)}\right)\right\rangle \mathrm{d} v=0,
$$

for any measures $f, g^{(1)}$ supported in $\mathbb{R}^{d} \times r \mathbb{S}^{d-1}$. Indeed, in that case the expression in 2.12 can be computed in terms of $f$, provided that we neglect the mass of $f^{(1)}$ at $\mathbb{R}^{d} \times\{0\}$ :

$$
\begin{aligned}
\int_{\mathbb{R}^{d}} \psi(v)\left\langle\operatorname{div}_{v} A_{f}\left(f^{(1)}\right)\right\rangle \mathrm{d} v= & \int_{\mathbb{R}^{d}} \psi\left\langle\operatorname{div}_{v} A_{f}\left\langle f^{(1)}\right\rangle\right\rangle \mathrm{d} v \\
& +\int_{\mathbb{R}^{d}} \psi\left\langle\operatorname{div}_{v} A_{f}\left[f^{(1)}-\left\langle f^{(1)}\right\rangle\right]\right\rangle \mathrm{d} v \\
= & \int_{\mathbb{R}^{d}} \psi(v)\left\langle\operatorname{div}_{v} A_{f}\left[f^{(1)}-\left\langle f^{(1)}\right\rangle\right]\right\rangle \mathrm{d} v .
\end{aligned}
$$

Let us concentrate now on the collision invariants of the average collision operator. Recall that the linearized of $\langle Q\rangle$, around a measure $F$ such that $\int_{\mathbb{R}^{d}} F \mathrm{~d} v>0$, writes

$$
\lim _{\varepsilon \searrow 0} \frac{\left\langle Q\left(F+\varepsilon F^{(1)}\right)\right\rangle-\langle Q(F)\rangle}{\varepsilon}=\left\langle\operatorname{div}_{v} A_{F}\left(F^{(1)}\right)\right\rangle
$$

where

$$
A_{F}\left(F^{(1)}\right)=\left[F^{(1)}(v-u[F])+\sigma \nabla_{v} F^{(1)}\right]-F \frac{\int_{\mathbb{R}^{d}} F^{(1)}\left(v^{\prime}-u[F]\right) \mathrm{d} v^{\prime}}{\int_{\mathbb{R}^{d}} F \mathrm{~d} v^{\prime}} .
$$

We search for functions $\psi=\psi(v)$ such that

$$
\int_{\mathbb{R}^{d}} \psi(v)\left\langle\operatorname{div}_{v} A_{F}\left(G^{(1)}\right)\right\rangle \mathrm{d} v=0,
$$

for any bounded measures $F, G^{(1)}$ supported in $r \mathbb{S}^{d-1}$. Actually, since we already know that the dominant term is a von Mises-Fisher distribution, it is enough to impose (2.13) only for $F=M_{l \Omega} \mathrm{d} \omega$, with $\lambda(l)=\frac{\sigma}{r^{2}} l$, for some given $\Omega \in \mathbb{S}^{d-1}$. Doing that, to any orientation $\Omega$, we associate a family of suitable pseudo-collision invariants, allowing us to determine the macroscopic equations satisfied by the moments $\rho, \Omega$. A similar construction was done in Ref. 48, baptized as generalized 
collision invariants. Even if our approach is not exactly the same as in Ref. 48, we will continue referring to them as generalized collision invariants. Notice that once we have determined $\psi$ such that $(2.13)$ is verified for any bounded measure $G^{(1)}$ supported in $r \mathbb{S}^{d-1}$, we need to check that (2.13) still holds true for any bounded measure, not necessarily supported in $r \mathbb{S}^{d-1}$, satisfying the constraint (1.8) (see Proposition [3.4 and Appendix C). The condition (2.13) should be understood in the following sense

$$
\int_{v \neq 0} \widetilde{\psi}\left(r \frac{v}{|v|}\right) \operatorname{div}_{v}\left\{A_{F}\left(G^{(1)}\right)\right\} \mathrm{d} v=0, \quad F=M_{l \Omega} \mathrm{d} \omega,
$$

for any $G^{(1)} \in \mathcal{M}_{b}\left(\mathbb{R}^{d}\right)$, supp $G^{(1)} \subset r \mathbb{S}^{d-1}$, that is

$$
\begin{gathered}
\int_{v \neq 0}\left\{-(v-u[F]) \cdot \nabla_{v}\left[\widetilde{\psi}\left(r \frac{v}{|v|}\right)\right]+\sigma \Delta_{v}\left[\widetilde{\psi}\left(r \frac{v}{|v|}\right)\right]\right\} G^{(1)} \mathrm{d} v \\
+\int_{v \neq 0} \frac{\int_{v^{\prime} \neq 0}\left(v^{\prime}-u[F]\right) G^{(1)} \mathrm{d} v^{\prime}}{\int_{\mathbb{R}^{d}} F \mathrm{~d} v^{\prime}} \cdot \nabla_{v}\left[\widetilde{\psi}\left(r \frac{v}{|v|}\right)\right] F \mathrm{~d} v=0,
\end{gathered}
$$

for $F=M_{l \Omega} \mathrm{d} \omega$ and any $G^{(1)} \in \mathcal{M}_{b}\left(\mathbb{R}^{d}\right)$, supp $G^{(1)} \subset r \mathbb{S}^{d-1}$. Taking into account the equalities

$$
\nabla_{v}\left[\widetilde{\psi}\left(r \frac{v}{|v|}\right)\right]=\nabla_{\omega} \widetilde{\psi}, \quad \Delta_{v}\left[\widetilde{\psi}\left(r \frac{v}{|v|}\right)\right]=\Delta_{\omega} \widetilde{\psi}, \quad|v|=r,
$$

the condition (2.14) becomes

$$
\left(\omega-u\left[M_{l \Omega}\right]\right) \cdot \nabla_{\omega} \widetilde{\psi}-\sigma \Delta_{\omega} \widetilde{\psi}=\left(\omega-u\left[M_{l \Omega}\right]\right) \cdot \frac{\int_{r \mathbb{S}^{d-1}} \nabla_{\omega^{\prime}} \widetilde{\psi} M_{l \Omega} \mathrm{d} \omega^{\prime}}{\int_{r \mathbb{S}^{d-1}} M_{l \Omega} \mathrm{d} \omega^{\prime}}=0 .
$$

\section{The Generalized Collision Invariants}

In this section, we concentrate on the resolution of the linear equation (2.15). If we introduce the vector

$$
W[\widetilde{\psi}]=\frac{\int_{r \mathbb{S}^{d-1}} \nabla_{\omega} \widetilde{\psi} M_{l \Omega}(\omega) \mathrm{d} \omega}{\int_{r \mathbb{S}^{d-1}} M_{l \Omega}(\omega) \mathrm{d} \omega}=\int_{r \mathbb{S}^{d-1}} \nabla_{\omega} \widetilde{\psi} M_{l \Omega}(\omega) \mathrm{d} \omega
$$

Eq. (2.15) becomes elliptic on $r \mathbb{S}^{d-1}$ and reads

$$
-\sigma \operatorname{div}_{\omega}\left(M_{l \Omega} \nabla_{\omega} \widetilde{\psi}\right)=M_{l \Omega}(\omega)\left(\omega-u\left[M_{l \Omega}\right]\right) \cdot W[\widetilde{\psi}] .
$$

Any solution of Eq. (3.1) will be called a generalized collision invariant of the average collision operator $\langle Q\rangle$.

The solvability of (3.1) requires that the integral of the right-hand side over $r \mathbb{S}^{d-1}$ vanishes, i.e.

$$
\int_{r \mathbb{S}^{d-1}} M_{l \Omega}(\omega)\left(\omega-u\left[M_{l \Omega}\right]\right) \cdot W[\widetilde{\psi}] \mathrm{d} \omega=0,
$$

which is true, by the definition of the mean velocity $u\left[M_{l \Omega}\right]$. But there is another compatibility condition to be fulfilled. Take any vector $W^{\prime} \in \mathbb{R}^{d}$ and multiply 
Eq. (3.1) by the scalar function $\omega \rightarrow W^{\prime} \cdot \omega$, whose gradient along $r \mathbb{S}^{d-1}$ is $\left(I_{d}-\frac{\omega \otimes \omega}{r^{2}}\right) W^{\prime}$. Integrating by parts yields

$$
\begin{aligned}
& \sigma \int_{r \mathbb{S}^{d-1}} M_{l \Omega}(\omega) \nabla_{\omega} \tilde{\psi} \mathrm{d} \omega \cdot W^{\prime} \\
& \quad=\int_{r \mathbb{S}^{d}-1} M_{l \Omega}(\omega)\left(\omega-u\left[M_{l \Omega}\right]\right) \otimes\left(\omega-u\left[M_{l \Omega}\right]\right) \mathrm{d} \omega: W[\widetilde{\psi}] \otimes W^{\prime},
\end{aligned}
$$

saying that $W[\widetilde{\psi}]$ is an eigenvector of the matrix

$$
\mathcal{M}_{l \Omega}:=\int_{r \mathbb{S}^{d-1}} M_{l \Omega}(\omega)\left(\omega-u\left[M_{l \Omega}\right]\right) \otimes\left(\omega-u\left[M_{l \Omega}\right]\right) \mathrm{d} \omega
$$

corresponding to the eigenvalue $\sigma$. The following lemma details the spectral properties of the matrix $\mathcal{M}_{l \Omega}$.

Lemma 3.1. For any $l \in \mathbb{R}_{+}$such that $\lambda(l)=\frac{\sigma}{r^{2}} l$, and $\Omega \in r \mathbb{S}^{d-1}$, the matrix $\mathcal{M}_{l \Omega}$ is symmetric, definite positive and:

$$
\begin{aligned}
& \mathcal{M}_{0 \Omega}=\frac{r^{2}}{d} I_{d}, \\
& \mathcal{M}_{l \Omega}=\left(r^{2}-(d-1) \sigma-|u|^{2}\right) \Omega \otimes \Omega+\sigma\left(I_{d}-\Omega \otimes \Omega\right), \quad l>0, \quad 0<\frac{\sigma}{r^{2}}<\frac{1}{d} .
\end{aligned}
$$

If $0<\frac{\sigma}{r^{2}}<\frac{1}{d}$, we have $r^{2}-(d-1) \sigma-|u|^{2}<\sigma$ and, in particular $\operatorname{ker}\left(\mathcal{M}_{l \Omega}-\sigma I_{d}\right)=$ $(\mathbb{R} \Omega)^{\perp}$.

Proof. Clearly $\mathcal{M}_{l \Omega}$ is symmetric and definite positive. The case $l=0$ is trivial, and we have $\mathcal{M}_{0 \Omega}=\frac{r^{2}}{d} I_{d}$. Assume now that $l>0$ and thus necessarily $\left.\frac{\sigma}{r^{2}} \in\right] 0, \frac{1}{d}$ [ cf. Proposition 1.5. We consider an orthonormal basis $\left\{E_{1}, \ldots, E_{d-1}, \Omega\right\}$. It is easily seen that

$$
\begin{aligned}
\mathcal{M}_{l \Omega}= & \int_{r \mathbb{S}^{d-1}}(\omega-u) \otimes \omega M_{l \Omega} \mathrm{d} \omega \\
= & \int_{r \mathbb{S}^{d-1}}\left[((\omega \cdot \Omega)-|u|) \Omega+\sum_{i=1}^{d-1}\left(\omega \cdot E_{i}\right) E_{i}\right] \otimes\left[(\omega \cdot \Omega) \Omega+\sum_{i=1}^{d-1}\left(\omega \cdot E_{i}\right) E_{i}\right] M_{l \Omega} \mathrm{d} \omega \\
= & \int_{r \mathbb{S}^{d-1}}((\omega \cdot \Omega)-|u|)(\omega \cdot \Omega) M_{l \Omega} \mathrm{d} \omega \Omega \otimes \Omega \\
& +\sum_{i=1}^{d-1} \int_{r \mathbb{S}^{d-1}}\left(\omega \cdot E_{i}\right)^{2} M_{l \Omega} \mathrm{d} \omega E_{i} \otimes E_{i} \\
= & \int_{r \mathbb{S}^{d-1}}\left((\omega \cdot \Omega)^{2}-|u|^{2}\right) M_{l \Omega} \mathrm{d} \omega \Omega \otimes \Omega \\
& +\int_{r \mathbb{S}^{d-1}} \frac{\left(r^{2}-(\omega \cdot \Omega)^{2}\right)}{d-1} M_{l \Omega} \mathrm{d} \omega\left(I_{d}-\Omega \otimes \Omega\right) .
\end{aligned}
$$


We show that

$$
\int_{r \mathbb{S}^{d-1}}(\omega \cdot \Omega)^{2} M_{l \Omega} \mathrm{d} \omega=r^{2}-(d-1) \sigma .
$$

This comes by the condition $\lambda(l)=\frac{\sigma}{r^{2}} l$ and integrations by parts

$$
\begin{aligned}
r^{2}-\int_{r \mathbb{S}^{d-1}}(\omega \cdot \Omega)^{2} M_{l \Omega} \mathrm{d} \omega & =\frac{r^{2} \int_{0}^{\pi} e^{l \cos \theta} \sin ^{d} \theta \mathrm{d} \theta}{\int_{0}^{\pi} e^{l \cos \theta} \sin ^{d-2} \theta \mathrm{d} \theta} \\
& =-\frac{r^{2}}{l} \frac{\int_{0}^{\pi} \frac{\mathrm{d}}{\mathrm{d} \theta} e^{l \cos \theta} \sin ^{d-1} \theta \mathrm{d} \theta}{\int_{0}^{\pi} e^{l \cos \theta} \sin ^{d-2} \theta \mathrm{d} \theta} \\
& =(d-1) \frac{r^{2}}{l} \frac{\int_{0}^{\pi} \cos \theta e^{l \cos \theta} \sin ^{d-2} \theta \mathrm{d} \theta}{\int_{0}^{\pi} e^{l \cos \theta} \sin ^{d-2} \theta \mathrm{d} \theta} \\
& =(d-1) \frac{r^{2}}{l} \lambda(l) \\
& =(d-1) \frac{r^{2}}{l} \frac{\sigma}{r^{2}} l=(d-1) \sigma .
\end{aligned}
$$

We deduce also that

$$
\int_{r \mathbb{S}^{d-1}}\left((\omega \cdot \Omega)^{2}-|u|^{2}\right) M_{l \Omega} \mathrm{d} \omega=r^{2}-(d-1) \sigma-|u|^{2}
$$

and therefore

$$
\mathcal{M}_{l \Omega}=\left(r^{2}-(d-1) \sigma-|u|^{2}\right) \Omega \otimes \Omega+\sigma\left(I_{d}-\Omega \otimes \Omega\right) .
$$

We claim that the biggest eigenvalue is $\sigma$, that is $r^{2}-(d-1) \sigma-|u|^{2}<\sigma$, or equivalently $r^{2}<d \sigma+|u|^{2}$. This is a consequence of Lemma 2.3 Indeed, since $l>0$, we know that

$$
\mu(l)=\frac{2 l}{\sqrt{d^{2}+4 l^{2}}+d}<\lambda(l)=\frac{\sigma}{r^{2}} l,
$$

implying that

$$
\sqrt{d^{2}+4 l^{2}}>\frac{2 r^{2}}{\sigma}-d>0, \quad \text { since } r^{2}>d \sigma
$$

or equivalently

$$
4 l^{2}>4 \frac{r^{4}}{\sigma^{2}}-4 d \frac{r^{2}}{\sigma}
$$

Replacing $l=\frac{|u| r}{\sigma}$ in the above inequality yields $r^{2}<d \sigma+|u|^{2}$.

The resolution of (2.15) follows immediately, thanks to Lemma 3.1. As (2.15) is linear and admits any constant function on $r \mathbb{S}^{d-1}$ as solution, we will work with zero mean solutions on $r \mathbb{S}^{d-1}$, that is $\int_{r \mathbb{S}^{d-1}} \widetilde{\psi}(\omega) \mathrm{d} \omega=0$. 
Proposition 3.1. Let $M_{l \Omega}$ be a von Mises-Fisher distribution, i.e. $\Omega \in \mathbb{S}^{d-1}$, $l \in \mathbb{R}_{+}, \lambda(l)=\frac{\sigma}{r^{2}} l$, and $E_{1}, \ldots, E_{d-1}$ be an orthonormal basis of $(\mathbb{R} \Omega)^{\perp}$.

(1) If $l=0$ and $\frac{\sigma}{r^{2}} \neq \frac{1}{d}$, then the only (zero mean) solution of (2.15) is the trivial one.

(2) If $l=0$ and $\frac{\sigma}{r^{2}}=\frac{1}{d}$, then the family of zero mean solutions for (2.15) is a linear space of dimension $d$. A basis is given by the functions $\widetilde{\psi}_{1}, \ldots, \widetilde{\psi}_{d}$ satisfying

$$
-\sigma \operatorname{div}_{\omega}\left(M_{0 \Omega} \nabla_{\omega} \widetilde{\psi}_{i}\right)=M_{0 \Omega}(\omega)\left(\omega \cdot E_{i}\right), \quad \int_{r \mathbb{S}^{d-1}} \widetilde{\psi}_{i}(\omega) \mathrm{d} \omega=0
$$

for $i \in\{1, \ldots, d\}$ and $E_{d}=\Omega$.

(3) If $0<\frac{\sigma}{r^{2}}<\frac{1}{d}, l>0, \lambda(l)=\frac{\sigma}{r^{2}} l$, then the family of zero mean solutions for (2.15) is a linear space of dimension $d-1$. A basis is given by the functions $\widetilde{\psi}_{1}, \ldots, \widetilde{\psi}_{d-1}$ satisfying

$$
-\sigma \operatorname{div}_{\omega}\left(M_{l \Omega} \nabla_{\omega} \widetilde{\psi}_{i}\right)=M_{l \Omega}(\omega)\left(\omega \cdot E_{i}\right), \quad \int_{r \mathbb{S}^{d-1}} \widetilde{\psi}_{i}(\omega) \mathrm{d} \omega=0,
$$

for $i \in\{1, \ldots, d-1\}$.

Proof. (1) Let $\widetilde{\psi}$ be a zero mean solution of 2.15$)$. Multiplying by $\left(\omega \cdot W^{\prime}\right)$, with $W^{\prime} \in \mathbb{R}^{d}$, and integrating by parts over $r \mathbb{S}^{d-1}$ yield

$$
\begin{aligned}
\sigma W[\widetilde{\psi}] \cdot W^{\prime} & =\sigma \int_{r \mathbb{S}^{d-1}} M_{0 \Omega} \nabla_{\omega} \widetilde{\psi} \cdot W^{\prime} \mathrm{d} \omega=\int_{r \mathbb{S}^{d-1}} M_{0 \Omega}(\omega-0) \cdot W[\widetilde{\psi}]\left(\omega \cdot W^{\prime}\right) \mathrm{d} \omega \\
& =\mathcal{M}_{0 \Omega} W[\widetilde{\psi}] \cdot W^{\prime}=\frac{r^{2}}{d} W[\widetilde{\psi}] \cdot W^{\prime} .
\end{aligned}
$$

Therefore $\left(\sigma-\frac{r^{2}}{d}\right) W[\widetilde{\psi}]=0$, implying that $W[\widetilde{\psi}]=0$ and

$$
-\operatorname{div}_{\omega}\left(M_{0 \Omega}(\omega) \nabla_{\omega} \widetilde{\psi}\right)=0 .
$$

We deduce that $\widetilde{\psi}$ is a constant, zero mean function on $r \mathbb{S}^{d-1}$, and thus $\widetilde{\psi}=0$.

(2) As $l=0$, then $\int_{r \mathbb{S}^{d-1}} \omega M_{0 \Omega}(\omega) \mathrm{d} \omega=u=0$. Therefore the right-hand sides in (3.2) are zero mean functions on $r \mathbb{S}^{d-1}$, and by Lax-Milgram lemma, the zero mean functions $\left(\widetilde{\psi}_{i}\right)_{1 \leq i \leq d}$ are well defined. Notice that these functions also solve (2.15). Indeed, after multiplication by $\left(\omega \cdot W^{\prime}\right)$, with $W^{\prime} \in \mathbb{R}^{d}$, and integration by parts we obtain, for any $i \in\{1, \ldots, d\}$ :

$$
\sigma \int_{r \mathbb{S}^{d-1}} \nabla_{\omega} \widetilde{\psi}_{i} \cdot W^{\prime} M_{0 \Omega} \mathrm{d} \omega=\int_{r \mathbb{S}^{d-1}}\left(\omega \cdot E_{i}\right)\left(\omega \cdot W^{\prime}\right) M_{0 \Omega} \mathrm{d} \omega=\mathcal{M}_{0 \Omega} E_{i} \cdot W^{\prime} .
$$

We deduce that

$$
\sigma \int_{r \mathbb{S}^{d-1}} M_{0 \Omega}(\omega) \nabla_{\omega} \widetilde{\psi}_{i} \mathrm{~d} \omega=\mathcal{M}_{0 \Omega} E_{i}=\frac{r^{2}}{d} E_{i}=\sigma E_{i}, \quad i \in\{1, \ldots, d\},
$$


which exactly says that $\left(\widetilde{\psi}_{i}\right)_{1 \leq i \leq d}$ solve (2.15). It is easily seen that the family $\left(\widetilde{\psi}_{i}\right)_{1 \leq i \leq d}$ is linearly independent: if $\sum_{i=1}^{d} c_{i} \widetilde{\psi}_{i}=0$, then by (3.4) one gets

$$
\sum_{i=1}^{d} c_{i} E_{i}=\sum_{i=1}^{d} c_{i} \int_{r \mathbb{S}^{d-1}} M_{0 \Omega}(\omega) \nabla_{\omega} \widetilde{\psi}_{i} \mathrm{~d} \omega=0
$$

implying that $c_{i}=0, i \in\{1, \ldots, d\}$. We show now that any zero mean solution $\widetilde{\psi}$ for 2.15) is a linear combination of $\left(\widetilde{\psi}_{i}\right)_{1 \leq i \leq d}$. Let $\left(c_{i}\right)_{1 \leq i \leq d}$ be the coordinates of the vector $W[\widetilde{\psi}]$ with respect to the basis $\left(E_{i}\right)_{1 \leq i \leq d}$ :

$$
W[\widetilde{\psi}]=\int_{r \mathbb{S}^{d-1}} M_{0 \Omega}(\omega) \nabla_{\omega} \tilde{\psi} \mathrm{d} \omega=\sum_{i=1}^{d} c_{i} E_{i} .
$$

We claim that $\widetilde{\psi}=\sum_{i=1}^{d} c_{i} \widetilde{\psi}_{i}$. Indeed, since $\widetilde{\psi}$ and $\sum_{i=1}^{d} c_{i} \widetilde{\psi}_{i}$ have zero mean, thanks to the uniqueness of zero mean solution, it is enough to check that $\sum_{i=1}^{d} c_{i} \widetilde{\psi}_{i}$ solves (3.1), with the right-hand side $M_{0 \Omega} \omega \cdot W[\widetilde{\psi}]$. Indeed, we have

$$
-\sigma \operatorname{div}_{\omega}\left(M_{0 \Omega} \nabla_{\omega} \sum_{i=1}^{d} c_{i} \widetilde{\psi}_{i}\right)=\sum_{i=1}^{d} c_{i} M_{0 \Omega}\left(\omega \cdot E_{i}\right)=M_{0 \Omega}(\omega-0) \cdot W[\widetilde{\psi}],
$$

implying that $\widetilde{\psi}=\sum_{i=1}^{d} c_{i} \widetilde{\psi}_{i}$.

(3) The arguments are similar. The solutions $\left(\widetilde{\psi}_{i}\right)_{1 \leq i \leq d-1}$ in (3.3) also solve (2.15), and are linearly independent. But for any solution $\widetilde{\psi}$ of 2.15), we have for any $W^{\prime} \in \mathbb{R}^{d}$ :

$$
\begin{aligned}
\sigma W[\widetilde{\psi}] \cdot W^{\prime} & =\sigma \int_{r \mathbb{S}^{d-1}} M_{l \Omega} \nabla_{\omega} \widetilde{\psi} \cdot W^{\prime} \mathrm{d} \omega \\
& =\int_{r \mathbb{S}^{d-1}} M_{l \Omega}\left(\omega-u\left[M_{l \Omega}\right]\right) \cdot W[\widetilde{\psi}]\left(\omega \cdot W^{\prime}\right) \mathrm{d} \omega \\
& =\mathcal{M}_{l \Omega} W[\widetilde{\psi}] \cdot W^{\prime} .
\end{aligned}
$$

Therefore $W[\widetilde{\psi}] \in \operatorname{ker}\left(\mathcal{M}_{l \Omega}-\sigma I_{d}\right)=(\mathbb{R} \Omega)^{\perp}=\operatorname{span}\left\{E_{1}, \ldots, E_{d-1}\right\}$ and we deduce that $\widetilde{\psi}=\sum_{i=1}^{d-1} c_{i} \widetilde{\psi}_{i}$, with $W[\widetilde{\psi}]=\sum_{i=1}^{d-1} c_{i} E_{i}$.

We focus now on the structure of the solutions of (2.15). This is a consequence of the symmetry of $M_{l \Omega}$, by rotations leaving invariant the orientation $\Omega$. We concentrate on the case $0<\frac{\sigma}{r^{2}}<\frac{1}{d}, \lambda(l)=\frac{\sigma}{r^{2}} l, l>0$.

Proposition 3.2. For any $W \in \mathbb{R}^{d}, W \cdot \Omega=0$, let us denote by $\widetilde{\psi}_{W}$ the unique solution of the problem

$$
-\sigma \operatorname{div}_{\omega}\left(M_{l \Omega} \nabla_{\omega} \widetilde{\psi}_{W}\right)=M_{l \Omega}(\omega-u) \cdot W=M_{l \Omega}(\omega \cdot W), \quad \int_{r \mathbb{S}^{d-1}} \widetilde{\psi}_{W} \mathrm{~d} \omega=0 .
$$

For any orthogonal transformation $\mathcal{O}$ of $\mathbb{R}^{d}$, leaving invariant the orientation $\Omega$, that is $\mathcal{O} \Omega=\Omega$, we have

$$
\widetilde{\psi}_{W}(\mathcal{O} \omega)=\widetilde{\psi}_{t} \mathcal{O}_{W}(\omega), \quad \omega \in r \mathbb{S}^{d-1} .
$$


Proof. We know that $\widetilde{\psi}_{W}$ is the minimum point of the functional

$$
J_{W}(z)=\frac{\sigma}{2} \int_{r \mathbb{S}^{d-1}} M_{l \Omega}\left|\nabla_{\omega} z\right|^{2} \mathrm{~d} \omega-\int_{r \mathbb{S}^{d-1}} M_{l \Omega}(\omega \cdot W) z(\omega) \mathrm{d} \omega
$$

on $z \in H^{1}\left(r \mathbb{S}^{d-1}\right), \int_{r \mathbb{S}^{d-1}} z(\omega) \mathrm{d} \omega=0$. It is easily seen that for any orthogonal transformation $\mathcal{O}$ of $\mathbb{R}^{d}$, and any function $z \in H^{1}\left(r \mathbb{S}^{d-1}\right), \int_{r \mathbb{S}^{d-1}} z(\omega) \mathrm{d} \omega=0$, we have

$$
z_{\mathcal{O}}:=z \circ \mathcal{O} \in H^{1}\left(r \mathbb{S}^{d-1}\right), \int_{r \mathbb{S}^{d-1}} z_{\mathcal{O}}(\omega) \mathrm{d} \omega=0
$$

and

$$
\left(\nabla_{\omega} z_{\mathcal{O}}\right)(\omega)={ }^{t} \mathcal{O}\left(\nabla_{\omega} z\right)(\mathcal{O} \omega), \quad \omega \in r \mathbb{S}^{d-1} .
$$

Moreover, for any $z \in H^{1}\left(r \mathbb{S}^{d-1}\right), \int_{r \mathbb{S}^{d-1}} z(\omega) \mathrm{d} \omega=0$, and any orthogonal transformation leaving invariant the orientation $\Omega$ we obtain

$$
\begin{aligned}
J_{t} \mathcal{O} W\left(z_{\mathcal{O}}\right)= & \left.\left.\frac{\sigma}{2} \int_{r \mathbb{S}^{d-1}} M_{l \Omega}(\omega)\right|^{t} \mathcal{O}\left(\nabla_{\omega} z\right)(\mathcal{O} \omega)\right|^{2} \mathrm{~d} \omega \\
& -\int_{r \mathbb{S}^{d-1}} M_{l \Omega}(\omega)\left(\omega \cdot{ }^{t} \mathcal{O} W\right) z(\mathcal{O} \omega) \mathrm{d} \omega \\
= & \frac{\sigma}{2} \int_{r \mathbb{S}^{d-1}} M_{l \Omega}(\mathcal{O} \omega)\left|\left(\nabla_{\omega} z\right)(\mathcal{O} \omega)\right|^{2} \mathrm{~d} \omega \\
& -\int_{r \mathbb{S}^{d-1}} M_{l \Omega}(\mathcal{O} \omega)(\mathcal{O} \omega \cdot W) z(\mathcal{O} \omega) \mathrm{d} \omega \\
= & \frac{\sigma}{2} \int_{r \mathbb{S}^{d-1}} M_{l \Omega}(\omega)\left|\nabla_{\omega} z(\omega)\right|^{2} \mathrm{~d} \omega-\int_{r \mathbb{S}^{d-1}} M_{l \Omega}(\omega)(\omega \cdot W) z(\omega) \mathrm{d} \omega \\
= & J_{W}(z) .
\end{aligned}
$$

Finally, one gets for any $z \in H^{1}\left(r \mathbb{S}^{d-1}\right), \int_{r \mathbb{S}^{d-1}} z(\omega) \mathrm{d} \omega=0$,

$$
J^{t}{ }_{\mathcal{O} W}\left(\widetilde{\psi}_{W} \circ \mathcal{O}\right)=J_{W}\left(\widetilde{\psi}_{W}\right) \leq J_{W}\left(z \circ{ }^{t} \mathcal{O}\right)=J_{t} \mathcal{O}(z),
$$

saying that $\widetilde{\psi}_{W} \circ \mathcal{O}=\widetilde{\psi}_{t} \mathcal{O} W$.

We claim that there is a function $\chi$ such that, for any $i \in\{1, \ldots, d-1\}$, the solution $\widetilde{\psi}_{i}$ writes

$$
\widetilde{\psi}_{i}(\omega)=\chi\left(\Omega \cdot \frac{\omega}{r}\right) c_{i}(\omega), \quad c_{i}(\omega)=\frac{\omega \cdot E_{i}}{\sqrt{r^{2}-(\Omega \cdot \omega)^{2}}}, \quad \omega \in r \mathbb{S}^{d-1} \backslash\{ \pm r \Omega\} .
$$

Lemma 3.2. We consider the vector field $F$ given by

$$
F(\omega)=\sum_{i=1}^{d-1} \widetilde{\psi}_{i}(\omega) E_{i}, \quad \omega \in r \mathbb{S}^{d-1} .
$$

Then the vector field $F$ does not depend on the orthonormal basis $\left\{E_{1}, \ldots, E_{d-1}\right\}$ of $(\mathbb{R} \Omega)^{\perp}$ and for any orthogonal transformation $\mathcal{O}$ of $\mathbb{R}^{d}$, preserving $\Omega$, we have

$$
F(\mathcal{O} \omega)=\mathcal{O} F(\omega), \quad \omega \in r \mathbb{S}^{d-1} .
$$


There is a function $\chi$ such that

$$
F(\omega)=\chi\left(\Omega \cdot \frac{\omega}{r}\right) \frac{\left(I_{d}-\Omega \otimes \Omega\right)(\omega)}{\sqrt{r^{2}-(\Omega \cdot \omega)^{2}}}, \quad \omega \in r \mathbb{S}^{d-1} \backslash\{ \pm r \Omega\}
$$

and thus, for any $i \in\{1, \ldots, d-1\}$, we have

$$
\widetilde{\psi}_{i}(\omega)=\chi\left(\Omega \cdot \frac{\omega}{r}\right) \frac{\omega \cdot E_{i}}{\sqrt{r^{2}-(\Omega \cdot \omega)^{2}}}, \quad \omega \in r \mathbb{S}^{d-1} \backslash\{ \pm r \Omega\} .
$$

Proof. Consider any other orthonormal basis $\left\{F_{1}, \ldots, F_{d-1}\right\}$ of $(\mathbb{R} \Omega)^{\perp}$. Thanks to the identities:

$$
\begin{aligned}
& E_{1} \otimes E_{1}+\cdots+E_{d-1} \otimes E_{d-1}+\Omega \otimes \Omega=I_{d}, \\
& F_{1} \otimes F_{1}+\cdots+F_{d-1} \otimes F_{d-1}+\Omega \otimes \Omega=I_{d},
\end{aligned}
$$

we obtain

$$
\begin{aligned}
\sum_{i=1}^{d-1} \widetilde{\psi}_{i} E_{i} & =\sum_{i=1}^{d-1} \widetilde{\psi}_{E_{i}} E_{i}=\sum_{i=1}^{d-1} \widetilde{\psi}_{\sum_{j=1}^{d-1}\left(E_{i} \cdot F_{j}\right) F_{j}} E_{i}=\sum_{i=1}^{d-1} \sum_{j=1}^{d-1}\left(E_{i} \cdot F_{j}\right) \widetilde{\psi}_{F_{j}} E_{i} \\
& =\sum_{j=1}^{d-1} \widetilde{\psi}_{F_{j}} \sum_{i=1}^{d-1}\left(E_{i} \cdot F_{j}\right) E_{i}=\sum_{j=1}^{d-1} \widetilde{\psi}_{F_{j}} F_{j} .
\end{aligned}
$$

Pick $\mathcal{O}$ any orthogonal transformation of $\mathbb{R}^{d}$, leaving invariant $\Omega$. For any $\omega \in$ $r \mathbb{S}^{d-1}$, we can write, by Proposition 3.2

$$
F(\mathcal{O} \omega)=\sum_{i=1}^{d-1} \widetilde{\psi}_{E_{i}}(\mathcal{O} \omega) E_{i}=\sum_{i=1}^{d-1} \widetilde{\psi}_{t} \mathcal{O E}_{i}(\omega) E_{i}=\mathcal{O} \sum_{i=1}^{d-1} \widetilde{\psi}_{t} \mathcal{O E}_{i}(\omega)^{t} \mathcal{O} E_{i}=\mathcal{O} F(\omega),
$$

where, in the last equality, we have used the independence of $F$ with respect to the orthonormal basis of $(\mathbb{R} \Omega)^{\perp}$. Take now $\omega \in r \mathbb{S}^{d-1} \backslash\{ \pm r \Omega\}$ and

$$
E=\frac{\left(I_{d}-\Omega \otimes \Omega\right) \omega}{\sqrt{r^{2}-(\Omega \cdot \omega)^{2}}} \text {. }
$$

Clearly $E \cdot \Omega=0,|E|=1$.

If $d=2$, as we know that $F(\omega) \cdot \Omega=0$, there is $\Lambda=\Lambda(\omega)$ such that

$$
F(\omega)=\Lambda(\omega) E=\Lambda(\omega) \frac{\left(I_{2}-\Omega \otimes \Omega\right) \omega}{\sqrt{r^{2}-(\Omega \cdot \omega)^{2}}} .
$$

If $d \geq 3$, take any unitary vector ${ }^{\perp} E$, orthogonal to $E$ and $\Omega$, and consider the symmetry $\mathcal{O}=I_{d}-2^{\perp} E \otimes{ }^{\perp} E$. The above orthogonal transformation leaves invariant $\Omega$, and thus, by the hypothesis, we know that $F\left(\mathcal{O} \omega^{\prime}\right)=\mathcal{O} F\left(\omega^{\prime}\right), \omega^{\prime} \in$ $r \mathbb{S}^{d-1}$. Observe that

$$
0={ }^{\perp} E \cdot E={ }^{\perp} E \cdot \frac{\omega-(\omega \cdot \Omega) \Omega}{\sqrt{r^{2}-(\Omega \cdot \omega)^{2}}}=\frac{{ }^{\perp} E \cdot \omega}{\sqrt{r^{2}-(\Omega \cdot \omega)^{2}}}, \quad \text { implying that } \mathcal{O} \omega=\omega,
$$


and thus

$$
F(\omega)=F(\mathcal{O} \omega)=\left(I_{d}-2{ }^{\perp} E \otimes{ }^{\perp} E\right) F(\omega)=F(\omega)-2\left(F(\omega) \cdot{ }^{\perp} E\right)^{\perp} E .
$$

We deduce that $F(\omega) \cdot{ }^{\perp} E=0$ for any vector ${ }^{\perp} E$, orthogonal to $E$ and $\Omega$. As $F(\omega) \cdot \Omega=0$, we deduce that $F(\omega)$ is orthogonal to any vector orthogonal to $E$, and thus there is $\Lambda=\Lambda(\omega)$ such that

$$
F(\omega)=\Lambda(\omega) E=\Lambda(\omega) \frac{\left(I_{d}-\Omega \otimes \Omega\right) \omega}{\sqrt{r^{2}-(\Omega \cdot \omega)^{2}}}, \quad \omega \in r \mathbb{S}^{d-1} \backslash\{ \pm r \Omega\} .
$$

We claim that $\Lambda(\omega)$ depends only on $\Omega \cdot \frac{\omega}{r}$. Indeed, for any $d \geq 2$, and any orthogonal transformation $\mathcal{O}$, such that $\mathcal{O} \Omega=\Omega$, we have $F(\mathcal{O} \omega)=\mathcal{O} F(\omega)$,

$$
\left(I_{d}-\Omega \otimes \Omega\right) \mathcal{O} \omega=\mathcal{O} \omega-(\Omega \cdot \mathcal{O} \omega) \Omega=\mathcal{O} \omega-(\Omega \cdot \omega) \mathcal{O} \Omega=\mathcal{O}\left(I_{d}-\Omega \otimes \Omega\right) \omega,
$$

for all $\omega \in r \mathbb{S}^{d-1} \backslash\{ \pm r \Omega\}$, and

$$
\begin{aligned}
\sqrt{r^{2}-(\Omega \cdot \mathcal{O} \omega)^{2}} & =\left|\left(I_{d}-\Omega \otimes \Omega\right) \mathcal{O} \omega\right|=\left|\mathcal{O}\left(I_{d}-\Omega \otimes \Omega\right) \omega\right| \\
& =\left|\left(I_{d}-\Omega \otimes \Omega\right) \omega\right|=\sqrt{r^{2}-(\Omega \cdot \omega)^{2}}
\end{aligned}
$$

implying that $\Lambda(\mathcal{O} \omega)=\Lambda(\omega), \omega \in r \mathbb{S}^{d-1} \backslash\{ \pm r \Omega\}$. Actually, the previous equality holds true for any $\omega \in r \mathbb{S}^{d-1}$, since $\mathcal{O} \Omega=\Omega$. We are done if we prove that $\Lambda(\omega)=$ $\Lambda\left(\omega^{\prime}\right)$ for any $\omega, \omega^{\prime} \in r \mathbb{S}^{d-1} \backslash\{ \pm r \Omega\}$ such that $\Omega \cdot \omega=\Omega \cdot \omega^{\prime}, \omega \neq \omega^{\prime}$. Consider the rotation $\mathcal{O}$ such that:

$$
\begin{aligned}
\mathcal{O} E & =E^{\prime},\left.\quad\left(\mathcal{O}-I_{d}\right)\right|_{\mathrm{span}\left\{E, E^{\prime}\right\}^{\perp}}=0, \\
E & =\frac{\left(I_{d}-\Omega \otimes \Omega\right) \omega}{\sqrt{r^{2}-(\Omega \cdot \omega)^{2}}}, \quad E^{\prime}=\frac{\left(I_{d}-\Omega \otimes \Omega\right) \omega^{\prime}}{\sqrt{r^{2}-\left(\Omega \cdot \omega^{\prime}\right)^{2}}} .
\end{aligned}
$$

Notice that the condition $\mathcal{O} E=E^{\prime}$ exactly says that $\mathcal{O} \omega=\omega^{\prime}$ and thus $\Lambda\left(\omega^{\prime}\right)=$ $\Lambda(\mathcal{O} \omega)=\Lambda(\omega)$. We deduce that there is a function $\chi$ such that $\Lambda(\omega)=\chi\left(\Omega \cdot \frac{\omega}{r}\right)$ and therefore

$$
\begin{aligned}
\sum_{i=1}^{d-1} \widetilde{\psi}_{i}(\omega) E_{i} & =F(\omega)=\chi\left(\Omega \cdot \frac{\omega}{r}\right) \frac{\left(I_{d}-\Omega \otimes \Omega\right) \omega}{\sqrt{r^{2}-(\Omega \cdot \omega)^{2}}} \\
& =\sum_{i=1}^{d-1} \chi\left(\Omega \cdot \frac{\omega}{r}\right) \frac{\omega \cdot E_{i}}{\sqrt{r^{2}-(\Omega \cdot \omega)^{2}}} E_{i},
\end{aligned}
$$

implying that

$$
\widetilde{\psi}_{i}(\omega)=\chi\left(\Omega \cdot \frac{\omega}{r}\right) \frac{\omega \cdot E_{i}}{\sqrt{r^{2}-(\Omega \cdot \omega)^{2}}}, \quad i \in\{1, \ldots, d-1\}, \quad \omega \in r \mathbb{S}^{d-1} \backslash\{ \pm r \Omega\} .
$$

Remark 3.1. In the case $d=2$, we take $E_{1}={ }^{\perp} \Omega, \omega=r\left(\cos \theta \Omega+\sin \theta^{\perp} \Omega\right)$ and therefore $\widetilde{\psi}_{1}$ writes

$$
\left.\widetilde{\psi}_{1}\left(r\left(\cos \theta \Omega+\sin \theta^{\perp} \Omega\right)\right)=\chi(\cos \theta) \operatorname{sign}(\sin \theta), \quad \theta \in\right]-\pi, 0[\cup] 0, \pi[.
$$


Clearly, the function $\bar{\psi}_{1}(\theta):=\widetilde{\psi}_{1}\left(r\left(\cos \theta \Omega+\sin \theta^{\perp} \Omega\right)\right)$ is odd (in particular $\left.\int_{r \mathbb{S}^{1}} \widetilde{\psi}_{1}(\omega) \mathrm{d} \omega=\int_{-\pi}^{\pi} \bar{\psi}_{1}(\theta) r \mathrm{~d} \theta=0\right)$ and the condition

$$
\int_{r \mathbb{S}^{1}}\left|\nabla_{\omega} \widetilde{\psi}_{1}\right|^{2} M_{l \Omega}(\omega) \mathrm{d} \omega<+\infty
$$

implies that $\int_{-\pi}^{\pi}\left|\partial_{\theta} \bar{\psi}_{1}\right|^{2} \mathrm{~d} \theta<+\infty$. Therefore $\bar{\psi}_{1}$ is continuous on $]-\pi, \pi[$, and thus $\chi(1)=0$. Notice that $\chi(-1)=0$ as well, since $\lim _{\theta \nearrow \pi} \bar{\psi}_{1}(\theta)=\widetilde{\psi}_{1}(-r \Omega)=$ $\lim _{\theta \searrow-\pi} \bar{\psi}_{1}(\theta)$.

Thanks to Lemma 3.2 in order to determine $\widetilde{\psi}_{i}, i \in\{1, \ldots, d-1\}$, we only need to solve for $\chi$. The idea is to analyze the behavior of the functionals $J_{E_{i}}$ on the set of functions $\Psi_{i, h}(\omega)=h\left(\Omega \cdot \frac{\omega}{r}\right) c_{i}(\omega), \omega \in r \mathbb{S}^{d-1}$. The notation $P_{\omega}$ stands for the orthogonal projection on the tangent space to $r \mathbb{S}^{d-1}$ at $\omega$, that is, $P_{\omega}=I_{d}-\frac{\omega \otimes \omega}{r^{2}}$.

Proposition 3.3. The function $\chi$ constructed in Lemma 3.2 solves the problem

$$
-\frac{\sigma}{r^{2}} \frac{\mathrm{d}}{\mathrm{d} c}\left\{e^{l c} \chi^{\prime}(c)\left(1-c^{2}\right)^{\frac{1}{2}}\right\}=r e^{l c}, \quad \chi(-1)=\chi(1)=0
$$

for all $c \in]-1,1[$, if $d=2$, and

$$
-\frac{\sigma}{r^{2}} \frac{\mathrm{d}}{\mathrm{d} c}\left\{e^{l c} \chi^{\prime}\left(1-c^{2}\right)^{\frac{d-1}{2}}\right\}+(d-2) \frac{\sigma}{r^{2}} e^{l c} \chi(c)\left(1-c^{2}\right)^{\frac{d-5}{2}}=r e^{l c}\left(1-c^{2}\right)^{\frac{d-2}{2}}
$$

for all $c \in]-1,1[$, if $d \geq 3$.

Proof. For any $i \in\{1, \ldots, d-1\}$, the gradient of $\Psi_{i, h}$ writes

$$
\nabla_{\omega} \Psi_{i, h}=h^{\prime}\left(\Omega \cdot \frac{\omega}{r}\right) c_{i}(\omega) \frac{P_{\omega} \Omega}{r}+h\left(\Omega \cdot \frac{\omega}{r}\right) \nabla_{\omega} c_{i}
$$

where

$$
\nabla_{\omega} c_{i}=\frac{P_{\omega} E_{i}}{\sqrt{r^{2}-(\omega \cdot \Omega)^{2}}}+\frac{\left(\omega \cdot E_{i}\right)(\omega \cdot \Omega)}{\left(r^{2}-(\omega \cdot \Omega)^{2}\right)^{3 / 2}} P_{\omega} \Omega .
$$

Therefore we obtain

$$
\begin{aligned}
\nabla_{\omega} \psi_{i, h}= & h^{\prime}\left(\Omega \cdot \frac{\omega}{r}\right) \frac{\omega \cdot E_{i}}{\sqrt{r^{2}-(\omega \cdot \Omega)^{2}}} \frac{P_{\omega} \Omega}{r} \\
& +\frac{h\left(\Omega \cdot \frac{\omega}{r}\right)}{\sqrt{r^{2}-(\omega \cdot \Omega)^{2}}}\left[P_{\omega} E_{i}+\frac{\left(\omega \cdot E_{i}\right)(\omega \cdot \Omega)}{r^{2}-(\omega \cdot \Omega)^{2}} P_{\omega} \Omega\right] .
\end{aligned}
$$

Notice that $P_{\omega} \Omega$ and $\nabla_{\omega} c_{i}$ are orthogonal, thanks to the equality $\left|P_{\omega} \Omega\right|^{2}=1-$ $\frac{(\omega \cdot \Omega)^{2}}{r^{2}}$. Indeed, we have

$$
\begin{aligned}
P_{\omega} \Omega & \cdot\left[P_{\omega} E_{i}+\frac{\left(\omega \cdot E_{i}\right)(\omega \cdot \Omega)}{r^{2}-(\omega \cdot \Omega)^{2}} P_{\omega} \Omega\right] \\
& =-\frac{\left(\omega \cdot E_{i}\right)(\omega \cdot \Omega)}{r^{2}}+\frac{\left(\omega \cdot E_{i}\right)(\omega \cdot \Omega)}{r^{2}-(\omega \cdot \Omega)^{2}}\left|P_{\omega} \Omega\right|^{2}=0 .
\end{aligned}
$$


Observe also that

$$
\left|\nabla_{\omega} c_{i}\right|^{2}=\frac{1}{r^{2}-(\omega \cdot \Omega)^{2}}\left[1-\frac{\left(\omega \cdot E_{i}\right)^{2}}{r^{2}-(\omega \cdot \Omega)^{2}}\right],
$$

implying that

$$
\begin{aligned}
\left|\nabla_{\omega} \Psi_{i, h}\right|^{2} & =\left(h^{\prime}\left(\Omega \cdot \frac{\omega}{r}\right) c_{i}(\omega)\right)^{2} \frac{\left|P_{\omega} \Omega\right|^{2}}{r^{2}}+\left(h\left(\Omega \cdot \frac{\omega}{r}\right)\right)^{2}\left|\nabla_{\omega} c_{i}\right|^{2} \\
& =\frac{\left(h^{\prime}\left(\Omega \cdot \frac{\omega}{r}\right)\right)^{2}\left(\omega \cdot E_{i}\right)^{2}}{r^{4}}+\frac{\left(h\left(\Omega \cdot \frac{\omega}{r}\right)\right)^{2}}{r^{2}-(\omega \cdot \Omega)^{2}}\left[1-\frac{\left(\omega \cdot E_{i}\right)^{2}}{r^{2}-(\omega \cdot \Omega)^{2}}\right] .
\end{aligned}
$$

Performing orthogonal changes of coordinates, which preserve $\Omega$, we deduce that the integrals $\int_{r \mathbb{S}^{d-1}}\left|\nabla_{\omega} \Psi_{i, h}\right|^{2} M_{l \Omega} \mathrm{d} \omega$ do not depend on $i \in\{1, \ldots, d-1\}$, and thus

$$
\begin{aligned}
\int_{r \mathbb{S}^{d-1}}\left|\nabla_{\omega} \Psi_{i, h}\right|^{2} M_{l \Omega} \mathrm{d} \omega= & \frac{1}{d-1} \int_{r \mathbb{S}^{d-1}} \frac{\left(h^{\prime}\left(\Omega \cdot \frac{\omega}{r}\right)\right)^{2}}{r^{4}}\left[r^{2}-(\omega \cdot \Omega)^{2}\right] M_{l \Omega} \mathrm{d} \omega \\
& +\frac{d-2}{d-1} \int_{r \mathbb{S}^{d-1}} \frac{\left(h\left(\Omega \cdot \frac{\omega}{r}\right)\right)^{2}}{r^{2}-(\omega \cdot \Omega)^{2}} M_{l \Omega} \mathrm{d} \omega .
\end{aligned}
$$

We also need to compute the linear part of the functional $J_{E_{i}}$ :

$$
\int_{r \mathbb{S}^{d-1}} M_{l \Omega}\left(\omega \cdot E_{i}\right) h\left(\Omega \cdot \frac{\omega}{r}\right) c_{i}(\omega) \mathrm{d} \omega=\int_{r \mathbb{S}^{d-1}} M_{l \Omega} \frac{h\left(\Omega \cdot \frac{\omega}{r}\right)}{d-1} \sqrt{r^{2}-(\omega \cdot \Omega)^{2}} \mathrm{~d} \omega .
$$

The expression of $J_{E_{i}}\left(\psi_{i, h}\right)$ follows by (3.7), (3.8):

$$
\begin{aligned}
J_{E_{i}}\left(\psi_{i, h}\right)= & \frac{\sigma}{2(d-1)} \int_{r \mathbb{S}^{d-1}} M_{l \Omega}\left(h^{\prime}\left(\Omega \cdot \frac{\omega}{r}\right)\right)^{2} \frac{r^{2}-(\Omega \cdot \omega)^{2}}{r^{4}} \mathrm{~d} \omega \\
& +\frac{\sigma}{2} \frac{d-2}{d-1} \int_{r \mathbb{S}^{d-1}} M_{l \Omega} \frac{\left(h\left(\Omega \cdot \frac{\omega}{r}\right)\right)^{2}}{r^{2}-(\omega \cdot \Omega)^{2}} \mathrm{~d} \omega \\
& -\frac{1}{d-1} \int_{r \mathbb{S}^{d-1}} M_{l \Omega} h\left(\Omega \cdot \frac{\omega}{r}\right) \sqrt{r^{2}-(\omega \cdot \Omega)^{2}} \mathrm{~d} \omega \\
= & \frac{\sigma}{2(d-1) r^{2}} \frac{\int_{0}^{\pi} e^{l \cos \theta}\left(h^{\prime}(\cos \theta)\right)^{2} \sin ^{d} \theta \mathrm{d} \theta}{\int_{0}^{\pi} e^{l \cos \theta} \sin ^{d-2} \theta \mathrm{d} \theta} \\
& +\frac{\sigma}{2} \frac{d-2}{d-1} \frac{\int_{0}^{\pi} e^{l \cos \theta}\left(\frac{h(\cos \theta)}{r \sin \theta}\right)^{2} \sin ^{d-2} \theta \mathrm{d} \theta}{\int_{0}^{\pi} e^{l \cos \theta} \sin ^{d-2} \theta \mathrm{d} \theta} \\
& -\frac{1}{d-1} \frac{\int_{0}^{\pi} e^{l \cos \theta} h(\cos \theta) r \sin ^{2} \sin ^{d-2} \theta \mathrm{d} \theta}{\int_{0}^{\pi} e^{l \cos \theta} \sin ^{d-2} \theta \mathrm{d} \theta} \\
= & \frac{J(h)}{(d-1) \pi \beta_{0}(l)},
\end{aligned}
$$


where $\pi \beta_{0}(l)=\int_{0}^{\pi} e^{l \cos \theta} \sin ^{d-2} \theta \mathrm{d} \theta$ and

$$
\begin{aligned}
J(h)= & \frac{\sigma}{2 r^{2}} \int_{-1}^{1} e^{l c}\left(h^{\prime}(c)\right)^{2}\left(1-c^{2}\right)^{\frac{d-1}{2}} \mathrm{~d} c+\frac{\sigma}{2} \frac{d-2}{r^{2}} \int_{-1}^{1} e^{l c}(h(c))^{2}\left(1-c^{2}\right)^{\frac{d-5}{2}} \mathrm{~d} c \\
& -r \int_{-1}^{1} e^{l c} h(c)\left(1-c^{2}\right)^{\frac{d-2}{2}} \mathrm{~d} c .
\end{aligned}
$$

We consider the Hilbert spaces

$$
H_{2}=\{h:]-1,1\left[\rightarrow \mathbb{R},\left(1-c^{2}\right)^{1 / 4} h^{\prime} \in L^{2}(]-1,1[), h(-1)=h(1)=0\right\}
$$

and

$$
H_{d}=\{h:]-1,1\left[\rightarrow \mathbb{R},\left(1-c^{2}\right)^{\frac{d-1}{4}} h^{\prime} \in L^{2}(]-1,1[),\left(1-c^{2}\right)^{\frac{d-5}{4}} h \in L^{2}(]-1,1[)\right\},
$$

for $d \geq 3$, endowed with the scalar products

$$
(g, h)_{2}=\int_{-1}^{1} g^{\prime}(c) h^{\prime}(c) \sqrt{1-c^{2}} \mathrm{~d} c, \quad g, h \in H_{2}
$$

and

$$
\begin{aligned}
(g, h)_{d}= & \int_{-1}^{1} g^{\prime}(c) h^{\prime}(c)\left(1-c^{2}\right)^{\frac{d-1}{2}} \mathrm{~d} c \\
& +\int_{-1}^{1} g(c) h(c)\left(1-c^{2}\right)^{\frac{d-5}{2}} \mathrm{~d} c, \quad g, h \in H_{d}, \quad \text { if } d \geq 3 .
\end{aligned}
$$

By Lemma 3.2, there is a function $\chi$ such that $\widetilde{\psi}_{i}=\chi\left(\Omega \cdot \frac{\omega}{r}\right) c_{i}(\omega), i \in\{1, \ldots$, $d-1\}$. We know that $\widetilde{\psi}_{i}, i \in\{1, \ldots, d-1\}$, minimize the functionals $J_{E_{i}}(z)$, with $z \in H^{1}\left(r \mathbb{S}^{d-1}\right), \int_{r \mathbb{S}^{d-1}} z(\omega) \mathrm{d} \omega=0$. In particular, for any $h \in H_{d}, d \geq 2$, we have

$$
J_{E_{i}}\left(\Psi_{i, h}\right) \geq J_{E_{i}}\left(\widetilde{\psi}_{i}\right), \quad \Psi_{i, h}(\omega)=h\left(\Omega \cdot \frac{\omega}{r}\right) c_{i}(\omega)
$$

implying that $\chi$, which belongs to $H_{d}$, is the solution of the minimization problem

$$
J(h) \geq J(\chi), \quad h \in H_{d} .
$$

Thanks to the Lax-Milgram lemma, we deduce that $\chi$ is the solution of the problem (3.5) if $d=2$, and (3.6) if $d \geq 3$.

Up to now, for a given equilibrium $F=M_{l \Omega} \mathrm{d} \omega$, we have determined the functions $\psi$ such that

$$
\int_{\mathbb{R}^{d}} \psi(v) \lim _{\varepsilon \searrow 0} \frac{\left\langle Q\left(F+\varepsilon G^{(1)}\right)\right\rangle-\langle Q(F)\rangle}{\varepsilon} \mathrm{d} v=0,
$$

for any bounded measure $G^{(1)}$, supported in $r \mathbb{S}^{d-1}$. But we need to control the linearization of $\langle Q\rangle$ around the equilibrium $F$ in the direction $F^{(1)}$, which is 
not necessarily supported in $r \mathbb{S}^{d-1}$. It happens that the constraint $\operatorname{div}_{v}\left\{F^{(1)}(\alpha-\right.$ $\left.\left.\beta|v|^{2}\right) v\right\}=Q(F)$, see (1.8), will guarantee that

$$
\int_{\mathbb{R}^{d}} \psi(v) \lim _{\varepsilon \searrow 0} \frac{\left\langle Q\left(F+\varepsilon F^{(1)}\right)\right\rangle-\langle Q(F)\rangle}{\varepsilon} \mathrm{d} v=\int_{\mathbb{R}^{d}} \psi(v)\left\langle\operatorname{div}_{v} A_{F}\left(F^{(1)}\right)\right\rangle \mathrm{d} v=0 .
$$

These computations are a little bit tedious and can be found in Appendix C

Proposition 3.4. Let $F=M_{l \Omega} \mathrm{d} \omega$ be a von Mises-Fisher distribution with $l>0$, and $F^{(1)}$ be a bounded measure (not charging a small neighborhood of 0, for simplifying), satisfying $\operatorname{div}_{v}\left\{F^{(1)}\left(\alpha-\beta|v|^{2}\right) v\right\}=Q(F)$. Then the linearized of $\langle Q\rangle$ around $F$ in the direction $F^{(1)}$ verifies

$\int_{\mathbb{R}^{d}} \widetilde{\psi}(v)\left\langle\operatorname{div}_{v} A_{F}\left(F^{(1)}\right)\right\rangle \mathrm{d} v=0, \quad$ for any generalized collision invariant $\widetilde{\psi}$ of $\langle Q\rangle$.

\section{The Limit Model}

We identify the model satisfied by the limit distribution $f=\lim _{\varepsilon \backslash 0} f^{\varepsilon}$. We already know that $f$ is a von Mises-Fisher distribution $f=\rho(t, x) M_{l \Omega(t, x)}(\omega) \mathrm{d} \omega$ with $\rho \geq$ $0, \Omega \in \mathbb{S}^{d-1}, l \geq 0, \lambda(l)=\frac{\sigma}{r^{2}} l$. If $\frac{\sigma}{r^{2}} \geq \frac{1}{d}$, then $l=0$ and $M_{l \Omega} \mathrm{d} \omega$ reduces to the isotropic measure on $r \mathbb{S}^{d-1}$, that is $f=\rho(t, x) \frac{\mathrm{d} \omega}{r^{d-1} \bar{\omega}_{d}}$, with zero mean velocity $u[f]=\int_{r \mathbb{S}^{d-1}} \omega \rho M_{l \Omega} \mathrm{d} \omega=0$. In this case, the continuity equation reduces to the trivial limit model $\partial_{t} \rho=0, t \in \mathbb{R}_{+}$. From now on, we assume that $\left.\frac{\sigma}{r^{2}} \in\right] 0, \frac{1}{d}[$, and we consider $l>0$ the unique solution for $\lambda(l)=\frac{\sigma}{r^{2}} l$, cf. Proposition 1.5. We are ready to justify the main result in Theorem 1.1 and the derivation of the $\mathrm{SOH}$ model (1.13)-(1.14).

Proof of Theorem 1.1. The continuity equation (1.13) comes from the continuity equation of (1.12):

$$
\partial_{t} \int_{\mathbb{R}^{d}} f \mathrm{~d} v+\operatorname{div}_{x} \int_{\mathbb{R}^{d}} f v \mathrm{~d} v=\lim _{\varepsilon \searrow 0}\left\{\partial_{t} \int_{\mathbb{R}^{d}} f^{\varepsilon} \mathrm{d} v+\operatorname{div}_{x} \int_{\mathbb{R}^{d}} f^{\varepsilon} v \mathrm{~d} v\right\}=0
$$

and the formula for the mean velocity of a von Mises-Fisher equilibrium

$$
u[f]=\int_{r \mathbb{S}^{d-1}} \omega \rho M_{l \Omega} \mathrm{d} \omega=\rho \frac{l \sigma}{r} \Omega=\rho \lambda(l) r \Omega .
$$

Equivalently, (1.13) is obtained by using the collision invariant $\widetilde{\psi}=1$. Equation (1.14) will follow, by using the $(d-1)$-dimensional linear space of collision invariants studied in Proposition 3.1, Revisiting the expansion (1.6), we obtain

$$
\partial_{t} f+\operatorname{div}_{x}(f v)+\operatorname{div}_{v}\left\{f^{(2)}\left(\alpha-\beta|v|^{2}\right) v\right\}=\operatorname{div}_{v}\left(A_{f}\left(f^{(1)}\right)\right),
$$


together with the constraints:

$$
\begin{aligned}
\operatorname{div}_{v}\left\{f\left(\alpha-\beta|v|^{2}\right) v\right\} & =0, \\
\operatorname{div}_{v}\left\{f^{(1)}\left(\alpha-\beta|v|^{2}\right) v\right\} & =Q(f) .
\end{aligned}
$$

The first constraint (4.2) says that, for any $(t, x) \in \mathbb{R}_{+} \times \mathbb{R}^{d}, \operatorname{supp} f(t, x) \subset\{0\} \cup$ $r \mathbb{S}^{d-1}$. Averaging the second constraint 4.3) leads to

$$
\langle Q(f)\rangle=\left\langle\operatorname{div}_{v}\left\{f^{(1)}\left(\alpha-\beta|v|^{2}\right) v\right\}\right\rangle=0
$$

and thus $f(t, x)=\rho(t, x) M_{l \Omega(t, x)}(\omega) \mathrm{d} \omega, \omega \in r \mathbb{S}^{d-1}$. Averaging (4.1) allows us to get rid of $f^{(2)}$ :

$$
\partial_{t}\langle f\rangle+\operatorname{div}_{x}\langle v f\rangle=\left\langle\operatorname{div}_{v} A_{f}\left(f^{(1)}\right)\right\rangle .
$$

In order to eliminate $f^{(1)}$ as well, we test (4.4) against the functions $\psi_{i}(v)=$ $\widetilde{\psi}_{i}\left(r \frac{v}{|v|}\right)$, where $\left(\widetilde{\psi}_{i}\right)_{1 \leq i \leq d-1}$ are the collision invariants constructed in Proposition 3.1. Indeed, by Proposition 3.4 we know that for any $i \in\{1, \ldots, d-1\}$ :

$$
\int_{v \neq 0} \widetilde{\psi}_{i}\left(r \frac{v}{|v|}\right)\left\langle\operatorname{div}_{v} A_{f}\left(f^{(1)}\right)\right\rangle \mathrm{d} v=\int_{v \neq 0} \widetilde{\psi}_{i}\left(r \frac{v}{|v|}\right) \operatorname{div}_{v} A_{f}\left(f^{(1)}\right) \mathrm{d} v=I\left[\widetilde{\psi}_{i}\right]=0
$$

and therefore

$$
\int_{r \mathbb{S}^{d-1}} \partial_{t}\left(\rho M_{l \Omega}\right) \widetilde{\psi}_{i} \mathrm{~d} \omega+\int_{r \mathbb{S}^{d-1}} \operatorname{div}_{x}\left(\rho M_{l \Omega} \omega\right) \widetilde{\psi}_{i}(\omega) \mathrm{d} \omega=0, \quad i \in\{1, \ldots, d-1\} .
$$

Let $\left\{E_{1}, \ldots, E_{d-1}, \Omega\right\}$ be an orthonormal basis and $\widetilde{\psi}_{1}, \ldots, \widetilde{\psi}_{d-1}$ be the solutions of the problems 3.3 . We recall that

$$
\sum_{i=1}^{d-1} \widetilde{\psi}_{i} E_{i}=F(\omega)=\chi\left(\Omega \cdot \frac{\omega}{r}\right) \frac{\left(I_{d}-\Omega \otimes \Omega\right) \frac{\omega}{r}}{\sqrt{1-\left(\Omega \cdot \frac{\omega}{r}\right)^{2}}} .
$$

Equation (4.5), written for $i \in\{1, \ldots, d-1\}$, says that

$$
\left(I_{d}-\Omega \otimes \Omega\right) \int_{r \mathbb{S}^{d-1}}\left[\partial_{t}\left(\rho M_{l \Omega}\right)+\operatorname{div}_{x}\left(\rho M_{l \Omega} \omega\right)\right] \frac{\chi\left(\frac{\omega}{r} \cdot \Omega\right)}{\sqrt{1-\left(\Omega \cdot \frac{\omega}{r}\right)^{2}}} \frac{\omega}{r} \mathrm{~d} \omega=0 .
$$

We need to compute the vectors:

$$
\begin{aligned}
U_{1} & =\int_{r \mathbb{S}^{d-1}} \partial_{t} \rho M_{l \Omega}(\omega) \frac{\chi\left(\frac{\omega}{r} \cdot \Omega\right)}{\sqrt{1-\left(\Omega \cdot \frac{\omega}{r}\right)^{2}}} \frac{\omega}{r} \mathrm{~d} \omega, \\
U_{2} & =\int_{r \mathbb{S}^{d-1}} \rho M_{l \Omega}(\omega) l \partial_{t} \Omega \cdot \frac{\omega}{r} \frac{\chi\left(\frac{\omega}{r} \cdot \Omega\right)}{\sqrt{1-\left(\Omega \cdot \frac{\omega}{r}\right)^{2}}} \frac{\omega}{r} \mathrm{~d} \omega, \\
U_{3} & =\int_{r \mathbb{S}^{d-1}} \omega \cdot \nabla_{x} \rho M_{l \Omega}(\omega) \frac{\chi\left(\frac{\omega}{r} \cdot \Omega\right)}{\sqrt{1-\left(\Omega \cdot \frac{\omega}{r}\right)^{2}}} \frac{\omega}{r} \mathrm{~d} \omega, \\
U_{4} & =\int_{r \mathbb{S}^{d-1}} l \rho \omega \cdot{ }^{t} \partial_{x} \Omega \frac{\omega}{r} M_{l \Omega}(\omega) \frac{\chi\left(\frac{\omega}{r} \cdot \Omega\right)}{\sqrt{1-\left(\Omega \cdot \frac{\omega}{r}\right)^{2}}} \frac{\omega}{r} \mathrm{~d} \omega
\end{aligned}
$$


and to impose

$$
\sum_{i=1}^{4}\left(I_{d}-\Omega \otimes \Omega\right) U_{i}=0 .
$$

Clearly, the first vector $U_{1}$ is parallel to $\Omega$, and thus

$$
\left(I_{d}-\Omega \otimes \Omega\right) U_{1}=0 .
$$

The treatment of the second and third vectors requires to compute

$$
\begin{aligned}
& \mathcal{A}:=\int_{r \mathbb{S}^{d-1}} \frac{\omega}{r} \otimes \frac{\omega}{r} M_{l \Omega}(\omega) \frac{\chi\left(\frac{\omega}{r} \cdot \Omega\right)}{\sqrt{1-\left(\Omega \cdot \frac{\omega}{r}\right)^{2}}} \mathrm{~d} \omega \\
& =\sum_{i=1}^{d-1} \int_{r \mathbb{S}^{d-1}} \frac{\left(\omega \cdot E_{i}\right)^{2}}{r^{2}} M_{l \Omega}(\omega) \frac{\chi\left(\frac{\omega}{r} \cdot \Omega\right)}{\sqrt{1-\left(\Omega \cdot \frac{\omega}{r}\right)^{2}}} \mathrm{~d} \omega E_{i} \otimes E_{i} \\
& +\int_{r \mathbb{S}^{d-1}} \frac{(\omega \cdot \Omega)^{2}}{r^{2}} M_{l \Omega}(\omega) \frac{\chi\left(\frac{\omega}{r} \cdot \Omega\right)}{\sqrt{1-\left(\Omega \cdot \frac{\omega}{r}\right)^{2}}} \mathrm{~d} \omega \Omega \otimes \Omega \\
& =\frac{1}{d-1} \int_{r \mathbb{S}^{d-1}}\left[1-\left(\Omega \cdot \frac{\omega}{r}\right)^{2}\right] M_{l \Omega}(\omega) \frac{\chi\left(\frac{\omega}{r} \cdot \Omega\right)}{\sqrt{1-\left(\Omega \cdot \frac{\omega}{r}\right)^{2}}} \mathrm{~d} \omega \sum_{i=1}^{d-1} E_{i} \otimes E_{i} \\
& +\int_{r \mathbb{S}^{d-1}} \frac{(\omega \cdot \Omega)^{2}}{r^{2}} M_{l \Omega}(\omega) \frac{\chi\left(\frac{\omega}{r} \cdot \Omega\right)}{\sqrt{1-\left(\Omega \cdot \frac{\omega}{r}\right)^{2}}} \mathrm{~d} \omega \Omega \otimes \Omega \\
& =\frac{\int_{0}^{\pi} \sin ^{2} \theta e^{l \cos \theta} \frac{\chi(\cos \theta)}{\sin \theta} \sin ^{d-2} \theta \mathrm{d} \theta}{\int_{0}^{\pi} e^{l \cos \theta} \sin ^{d-2} \theta \mathrm{d} \theta} \frac{I_{d}-\Omega \otimes \Omega}{d-1} \\
& +\frac{\int_{0}^{\pi} \cos ^{2} \theta e^{l \cos \theta} \frac{\chi(\cos \theta)}{\sin \theta} \sin ^{d-2} \theta \mathrm{d} \theta}{\int_{0}^{\pi} e^{l \cos \theta} \sin ^{d-2} \theta \mathrm{d} \theta} \Omega \otimes \Omega .
\end{aligned}
$$

We obtain, thanks to the identity $\partial_{t} \Omega \cdot \Omega=\frac{1}{2} \partial_{t}|\Omega|^{2}=0$,

$$
\begin{aligned}
\left(I_{d}-\Omega \otimes \Omega\right) U_{2} & =\left(I_{d}-\Omega \otimes \Omega\right) \rho l \mathcal{A} \partial_{t} \Omega \\
& =\frac{\rho l}{d-1} \frac{\int_{0}^{\pi} e^{l \cos \theta} \chi(\cos \theta) \sin ^{d-1} \theta \mathrm{d} \theta}{\int_{0}^{\pi} e^{l \cos \theta} \sin ^{d-2} \theta \mathrm{d} \theta} \partial_{t} \Omega
\end{aligned}
$$

and

$$
\begin{aligned}
\left(I_{d}-\Omega \otimes \Omega\right) U_{3} & =r\left(I_{d}-\Omega \otimes \Omega\right) \mathcal{A} \nabla_{x} \rho \\
& =\frac{r}{d-1} \frac{\int_{0}^{\pi} e^{l \cos \theta} \chi(\cos \theta) \sin ^{d-1} \theta \mathrm{d} \theta}{\int_{0}^{\pi} e^{l \cos \theta} \sin ^{d-2} \theta \mathrm{d} \theta}\left(I_{d}-\Omega \otimes \Omega\right) \nabla_{x} \rho .
\end{aligned}
$$

We concentrate now on the last vector $U_{4}$. Observe that

$$
\begin{aligned}
\left(I_{d}\right. & -\Omega \otimes \Omega) U_{4} \\
& =r \rho l \int_{r \mathbb{S}^{d-1}} \frac{\omega}{r} \otimes \frac{\omega}{r}: \partial_{x} \Omega M_{l \Omega}(\omega) \frac{\chi\left(\frac{\omega}{r} \cdot \Omega\right)}{\sqrt{1-\left(\Omega \cdot \frac{\omega}{r}\right)^{2}}} \sum_{i=1}^{d-1}\left(E_{i} \cdot \frac{\omega}{r}\right) E_{i} \mathrm{~d} \omega
\end{aligned}
$$


and for any $i \in\{1, \ldots, d-1\}$ :

$$
\begin{aligned}
\int_{r \mathbb{S}^{d-1}} & \frac{\omega}{r} \otimes \frac{\omega}{r}: \partial_{x} \Omega M_{l \Omega}(\omega) \frac{\chi\left(\frac{\omega}{r} \cdot \Omega\right)}{\sqrt{1-\left(\Omega \cdot \frac{\omega}{r}\right)^{2}}}\left(E_{i} \cdot \frac{\omega}{r}\right) \mathrm{d} \omega \\
= & \int_{r \mathbb{S}^{d-1}} \frac{\left(\omega \cdot E_{i}\right)^{2}(\omega \cdot \Omega)}{r^{3}} M_{l \Omega}(\omega) \frac{\chi\left(\frac{\omega}{r} \cdot \Omega\right)}{\sqrt{1-\left(\Omega \cdot \frac{\omega}{r}\right)^{2}}} \mathrm{~d} \omega \\
& \times\left[E_{i} \otimes \Omega: \partial_{x} \Omega+\Omega \otimes E_{i}: \partial_{x} \Omega\right] \\
= & \int_{r \mathbb{S}^{d-1}} \frac{1-\left(\Omega \cdot \frac{\omega}{r}\right)^{2}}{d-1} \frac{(\omega \cdot \Omega)}{r} M_{l \Omega}(\omega) \frac{\chi\left(\frac{\omega}{r} \cdot \Omega\right)}{\sqrt{1-\left(\Omega \cdot \frac{\omega}{r}\right)^{2}}} \mathrm{~d} \omega \\
& \times\left[E_{i} \otimes \Omega: \partial_{x} \Omega+\Omega \otimes E_{i}: \partial_{x} \Omega\right] \\
= & \frac{1}{d-1} \frac{\int_{0}^{\pi} \sin ^{2} \theta \cos \theta e^{l \cos \theta} \frac{\chi(\cos \theta)}{\sin \theta} \sin ^{d-2} \theta \mathrm{d} \theta}{\int_{0}^{\pi} e^{l \cos \theta} \sin ^{d-2} \theta \mathrm{d} \theta}\left(\partial_{x} \Omega \Omega \cdot E_{i}+{ }^{t} \partial_{x} \Omega \Omega \cdot E_{i}\right) .
\end{aligned}
$$

Thanks to the formula ${ }^{t} \partial_{x} \Omega \Omega=\frac{1}{2} \nabla_{x}|\Omega|^{2}=0$, we obtain

$$
\begin{aligned}
\left(I_{d}-\Omega \otimes \Omega\right) U_{4} & =\frac{r \rho l}{d-1} \frac{\int_{0}^{\pi} \cos \theta e^{l \cos \theta} \chi(\cos \theta) \sin ^{d-1} \theta \mathrm{d} \theta}{\int_{0}^{\pi} e^{l \cos \theta} \sin ^{d-2} \theta \mathrm{d} \theta} \sum_{i=1}\left(\partial_{x} \Omega \Omega \cdot E_{i}\right) E_{i} \\
& =\frac{r \rho l}{d-1} \frac{\int_{0}^{\pi} \cos \theta e^{l \cos \theta} \chi(\cos \theta) \sin ^{d-1} \theta \mathrm{d} \theta}{\int_{0}^{\pi} e^{l \cos \theta} \sin ^{d-2} \theta \mathrm{d} \theta}\left(I_{d}-\Omega \otimes \Omega\right) \partial_{x} \Omega \Omega \\
& =\frac{r \rho l}{d-1} \frac{\int_{0}^{\pi} \cos \theta e^{l \cos \theta} \chi(\cos \theta) \sin ^{d-1} \theta \mathrm{d} \theta}{\int_{0}^{\pi} e^{l \cos \theta} \sin ^{d-2} \theta \mathrm{d} \theta} \partial_{x} \Omega \Omega
\end{aligned}
$$

The evolution equation for the orientation $\Omega$ comes now by collecting 4.6) -4.10) to get

$$
\begin{array}{r}
\frac{\rho l \partial_{t} \Omega+r\left(I_{d}-\Omega \otimes \Omega\right) \nabla_{x} \rho}{d-1} \frac{\int_{0}^{\pi} e^{l \cos \theta} \chi(\cos \theta) \sin ^{d-1} \theta \mathrm{d} \theta}{\int_{0}^{\pi} e^{l \cos \theta} \sin ^{d-2} \theta \mathrm{d} \theta} \\
+\frac{r \rho l}{d-1} \frac{\int_{0}^{\pi} \cos \theta e^{l \cos \theta} \chi(\cos \theta) \sin ^{d-1} \theta \mathrm{d} \theta}{\int_{0}^{\pi} e^{l \cos \theta} \sin ^{d-2} \theta \mathrm{d} \theta} \partial_{x} \Omega \Omega=0
\end{array}
$$

which also rewrites as

$$
\partial_{t} \Omega+r \frac{\int_{0}^{\pi} \cos \theta e^{l \cos \theta} \chi(\cos \theta) \sin ^{d-1} \theta \mathrm{d} \theta}{\int_{0}^{\pi} e^{l \cos \theta} \chi(\cos \theta) \sin ^{d-1} \theta \mathrm{d} \theta}\left(\Omega \cdot \nabla_{x}\right) \Omega+\frac{r}{l}\left(I_{d}-\Omega \otimes \Omega\right) \frac{\nabla_{x} \rho}{\rho}=0 .
$$

Remark 4.1. Taking the scalar product of Eq. (1.14) with $\Omega$, we obtain

$$
\frac{1}{2} \partial_{t}|\Omega|^{2}+\frac{k_{d} r}{2}\left(\Omega \cdot \nabla_{x}\right)|\Omega|^{2}=0, \quad(t, x) \in \mathbb{R}_{+} \times \mathbb{R}^{d},
$$

implying that $|\Omega(t, x)|=1,(t, x) \in \mathbb{R}_{+} \times \mathbb{R}^{d}$, provided that $|\Omega(0, x)|=1, x \in \mathbb{R}^{d}$. 


\section{Appendix A. Integration by Parts on Spheres}

Proof of Lemma 2.1. We pick a function $\eta \in C_{c}^{1}(] r_{1}, r_{2}[)$ and observe that

$$
\operatorname{div}_{v}\{\eta(|v|) A(v)\}=\eta^{\prime}(|v|) \frac{v}{|v|} \cdot A(v)+\eta(|v|)\left(\operatorname{div}_{v} A\right)(v), \quad v \in \mathcal{O} .
$$

Integrating with respect to $v$ over $\mathcal{O}$ leads to

$$
\begin{aligned}
0 & =\int_{\mathcal{O}} \operatorname{div}_{v}\{\eta(|v|) A(v)\} \mathrm{d} v=\int_{\mathcal{O}} \eta^{\prime}(|v|) \frac{v}{|v|} \cdot A(v) \mathrm{d} v+\int_{\mathcal{O}} \eta(|v|)\left(\operatorname{div}_{v} A\right)(v) \mathrm{d} v \\
& =\int_{r_{1}}^{r_{2}} \eta^{\prime}(t) \int_{|\omega|=1} \omega \cdot A(t \omega) t^{d-1} \mathrm{~d} \omega \mathrm{d} t+\int_{r_{1}}^{r_{2}} \eta(t) \int_{|\omega|=1}\left(\operatorname{div}_{v} A\right)(t \omega) t^{d-1} \mathrm{~d} \omega \mathrm{d} t \\
& =\int_{r_{1}}^{r_{2}} \eta(t)\left[-\frac{\mathrm{d}}{\mathrm{d} t} \int_{|\omega|=1} \omega \cdot A(t \omega) t^{d-1} \mathrm{~d} \omega+\int_{|\omega|=1}\left(\operatorname{div}_{v} A\right)(t \omega) t^{d-1} \mathrm{~d} \omega\right] \mathrm{d} t .
\end{aligned}
$$

We deduce that

$$
\begin{aligned}
\int_{|\omega|=t}\left(\operatorname{div}_{v} A\right)(\omega) \mathrm{d} \omega & =\frac{\mathrm{d}}{\mathrm{d} t} \int_{|\omega|=1} \omega \cdot A(t \omega) t^{d-1} \mathrm{~d} \omega \\
& =\int_{|\omega|=1}\left\{\omega \cdot \partial_{v} A(t \omega) \omega t^{d-1}+\omega \cdot A(t \omega)(d-1) t^{d-2}\right\} \mathrm{d} \omega \\
& =\int_{|\omega|=t}\left\{\frac{\omega \otimes \omega}{t^{2}}: \partial_{v} A(\omega)+\frac{(d-1) \omega}{t^{2}} \cdot A(\omega)\right\} \mathrm{d} \omega .
\end{aligned}
$$

Assume now that $A(v) \cdot v=0, v \in \mathcal{O}$. Taking the gradient with respect to $v$ yields ${ }^{t} \partial_{v} A(v) v+A(v)=0$ implying $\partial_{v} A(v): v \otimes v=-A(v) \cdot v=0, v \in \mathcal{O}$. In this case (2.1) reduces to (2.2). The formula in (2.3) follows easily by applying (2.2) with the field $v \rightarrow \chi(v) A(v)$.

\section{Appendix B. Differential Operators on Spheres}

Proof of Lemma 2.2. (1) Pick a point $\omega \in r \mathbb{S}^{d-1}$ and a tangent vector $X \in$ $T_{\omega}\left(r \mathbb{S}^{d-1}\right)$. Let $\left.\gamma:\right]-\varepsilon, \varepsilon\left[\rightarrow r \mathbb{S}^{d-1}\right.$ be a smooth curve such that $\gamma(0)=\omega, \gamma^{\prime}(0)=X$. Then we have

$$
\begin{aligned}
\nabla_{\omega} \widetilde{\psi} \cdot X & =\mathrm{d} \widetilde{\psi}_{\omega}(X)=\left.\frac{\mathrm{d}}{\mathrm{d} t}\right|_{t=0} \widetilde{\psi}(\gamma(t))=\left.\frac{\mathrm{d}}{\mathrm{d} t}\right|_{t=0} \psi(\gamma(t)) \\
& =\widetilde{\nabla_{v} \psi}(\omega) \cdot X=\left(I_{d}-\frac{\omega \otimes \omega}{r^{2}}\right) \widetilde{\nabla_{v} \psi}(\omega) \cdot X,
\end{aligned}
$$

saying that

$$
\nabla_{\omega} \widetilde{\psi}-\left(I_{d}-\frac{\omega \otimes \omega}{r^{2}}\right) \widetilde{\nabla_{v} \psi} \in T_{\omega}\left(r \mathbb{S}^{d-1}\right) \cap\left(T_{\omega}\left(r \mathbb{S}^{d-1}\right)\right)^{\perp}=\{0\} .
$$

Therefore we deduce that $\nabla_{\omega} \widetilde{\psi}=\left(I_{d}-\frac{\omega \otimes \omega}{r^{2}}\right) \widetilde{\nabla_{v} \psi}$. 
(2) For any $\omega_{t} \in t \mathbb{S}^{d-1}$ and $X \in T_{\omega_{t}}\left(t \mathbb{S}^{d-1}\right)$, pick a smooth curve $\left.\gamma:\right]-\varepsilon, \varepsilon\left[\rightarrow t \mathbb{S}^{d-1}\right.$ such that $\gamma(0)=\omega_{t}, \gamma^{\prime}(0)=X$. Therefore we have

$$
\nabla_{\omega_{t}} \widetilde{\psi}^{t}\left(\omega_{t}\right) \cdot X=\frac{\mathrm{d}}{\left.\mathrm{d} s\right|_{s=0}} \psi(\gamma(s))=\frac{\mathrm{d}}{\left.\mathrm{d} s\right|_{s=0}} \widetilde{\psi}\left(r \frac{\gamma(s)}{t}\right)=\nabla_{\omega} \widetilde{\psi}\left(r \frac{\omega_{t}}{t}\right) \cdot \frac{r}{t} X
$$

saying that $\left(\nabla_{\omega_{t}} \widetilde{\psi}^{t}\right)\left(\omega_{t}\right)=\frac{r}{t}\left(\nabla_{\omega} \widetilde{\psi}\right)\left(r \frac{\omega_{t}}{t}\right)$. Actually the function $\psi$ has only tangent gradient (to the spheres), and thus

$$
\left(\nabla_{v} \psi\right)\left(\omega_{t}\right)=\left(\nabla_{\omega_{t}} \widetilde{\psi}^{t}\right)\left(\omega_{t}\right)=\frac{r}{t}\left(\nabla_{\omega} \widetilde{\psi}\right)\left(r \frac{\omega_{t}}{t}\right), \quad\left|\omega_{t}\right|=t
$$

(3) Consider a $C^{1}$-function $\widetilde{\psi}$ on $r \mathbb{S}^{d-1}$ and $\psi$ a $C^{1}$-extension of $\widetilde{\psi}$ on $\mathcal{O}$. By Lemma 2.1, we know that

$$
\int_{|\omega|=r} \widetilde{\nabla_{v} \psi}(\omega) \cdot \widetilde{\xi}(\omega) \mathrm{d} \omega+\int_{|\omega|=r} \widetilde{\psi}(\omega) \widetilde{\operatorname{div}_{v} \xi}(\omega) \mathrm{d} \omega=0 .
$$

But, by the previous statement, we can write

$$
\begin{aligned}
\widetilde{\nabla_{v} \psi}(\omega) \cdot \widetilde{\xi}(\omega) & =\widetilde{\nabla_{v} \psi}(\omega) \cdot\left(I_{d}-\frac{\omega \otimes \omega}{r^{2}}\right) \widetilde{\xi}(\omega)=\left(I_{d}-\frac{\omega \otimes \omega}{r^{2}}\right) \widetilde{\nabla_{v} \psi}(\omega) \cdot \tilde{\xi}(\omega) \\
& =\nabla_{\omega} \widetilde{\psi}(\omega) \cdot \widetilde{\xi}(\omega) .
\end{aligned}
$$

Combining (B.1), (B.2) yields

$$
\begin{aligned}
\int_{|\omega|=r} \widetilde{\psi}(\omega) \operatorname{div}_{\omega} \widetilde{\xi}(\omega) \mathrm{d} \omega & =-\int_{|\omega|=r} \nabla_{\omega} \widetilde{\psi}(\omega) \cdot \widetilde{\xi}(\omega) \mathrm{d} \omega \\
& =\int_{|\omega|=r} \widetilde{\psi}(\omega) \widetilde{\operatorname{div}_{v} \xi}(\omega) \mathrm{d} \omega, \quad \widetilde{\psi} \in C^{1}\left(r \mathbb{S}^{d-1}\right),
\end{aligned}
$$

implying that $\operatorname{div}_{\omega} \widetilde{\xi}=\widetilde{\operatorname{div}_{v} \xi}$.

(4) Consider $\widetilde{\xi}=\widetilde{\xi}(\omega)$ a $C^{1}$-tangent vector field on $r \mathbb{S}^{d-1}$ and $\xi(v)=\widetilde{\xi}\left(r \frac{v}{|v|}\right), v \in$ $\mathbb{R}^{d} \backslash\{0\}$. We have $\xi(v) \cdot v=0, v \in \mathbb{R}^{d} \backslash\{0\}$, and for any $t>0$ :

$$
\left(\operatorname{div}_{v} \xi\right)\left(\omega_{t}\right)=\left(\operatorname{div}_{\omega_{t}} \widetilde{\xi}^{t}\right)\left(\omega_{t}\right)=\frac{r}{t}\left(\operatorname{div}_{\omega} \widetilde{\xi}\right)\left(r \frac{\omega_{t}}{t}\right), \quad \omega_{t} \in t \mathbb{S}^{d-1}
$$

The first equality comes by the third statement of Lemma 2.2 In order to check the second equality, pick a $C^{1}$-function $\widetilde{\psi}^{t}$ on $t \mathbb{S}^{d-1}$ and consider the function $\widetilde{\psi}(\omega)=\widetilde{\psi}^{t}(t \omega / r), \omega \in r \mathbb{S}^{d-1}$. We have

$$
\nabla_{\omega} \widetilde{\psi}(\omega)=\frac{t}{r}\left(\nabla_{\omega_{t}} \widetilde{\psi}^{t}\right)\left(t \frac{\omega}{r}\right)
$$


and thus

$$
\begin{aligned}
-\int_{\left|\omega_{t}\right|=t}\left(\operatorname{div}_{\omega_{t}} \widetilde{\xi}^{t}\right)\left(\omega_{t}\right) \widetilde{\psi}^{t}\left(\omega_{t}\right) \mathrm{d} \omega_{t} & =\int_{\left|\omega_{t}\right|=t} \widetilde{\xi}^{t}\left(\omega_{t}\right) \cdot \nabla_{\omega_{t}} \widetilde{\psi}^{t}\left(\omega_{t}\right) \mathrm{d} \omega_{t} \\
& =\int_{|\omega|=r} \xi\left(t \frac{\omega}{r}\right) \cdot\left(\nabla_{\omega_{t}} \widetilde{\psi}^{t}\right)\left(t \frac{\omega}{r}\right)\left(\frac{t}{r}\right)^{d-1} \mathrm{~d} \omega \\
& =\int_{|\omega|=r} \widetilde{\xi}(\omega) \cdot \nabla_{\omega} \widetilde{\psi}(\omega)\left(\frac{t}{r}\right)^{d-2} \mathrm{~d} \omega \\
& =-\int_{|\omega|=r}\left(\operatorname{div}_{\omega} \widetilde{\xi}\right)(\omega) \widetilde{\psi}(\omega)\left(\frac{t}{r}\right)^{d-2} \mathrm{~d} \omega \\
& =-\int_{\left|\omega_{t}\right|=t} \frac{r}{t}\left(\operatorname{div}_{\omega} \widetilde{\xi}\right)\left(r \frac{\omega_{t}}{t}\right) \widetilde{\psi}^{t}\left(\omega_{t}\right) \mathrm{d} \omega_{t} .
\end{aligned}
$$

We deduce that $\left(\operatorname{div}_{\omega_{t}} \xi\right)\left(\omega_{t}\right)=\frac{r}{t}\left(\operatorname{div}_{\omega} \widetilde{\xi}\right)\left(r \omega_{t} / t\right)$ for any $\omega_{t} \in t \mathbb{S}^{d-1}$.

\section{Appendix C. Collision Invariants and Linearization of $\langle Q\rangle$}

Proof of Proposition 3.4. Consider a collision invariant $\widetilde{\psi}$, and let us compute

$$
I[\widetilde{\psi}]:=\int_{v \neq 0} \widetilde{\psi}\left(r \frac{v}{|v|}\right) \operatorname{div}_{v} A_{F}\left(F^{(1)}\right) \mathrm{d} v
$$

that is

$$
\begin{aligned}
I[\widetilde{\psi}]= & \int_{v \neq 0}\left\{-(v-u[F]) \cdot \nabla_{v}\left[\widetilde{\psi}\left(r \frac{v}{|v|}\right)\right]+\sigma \Delta_{v}\left[\widetilde{\psi}\left(r \frac{v}{|v|}\right)\right]\right\} F^{(1)} \mathrm{d} v \\
& +\int_{v \neq 0}(v-u[F]) \cdot \frac{\int_{v^{\prime} \neq 0} \nabla_{v^{\prime}}\left[\widetilde{\psi}\left(r \frac{v^{\prime}}{\left|v^{\prime}\right|}\right)\right] F \mathrm{~d} v^{\prime}}{\int_{\mathbb{R}^{d}} F \mathrm{~d} v^{\prime}} F^{(1)} \mathrm{d} v .
\end{aligned}
$$

We consider the application

$$
\begin{aligned}
\chi(v)= & -(v-u[F]) \cdot \nabla_{v}\left[\widetilde{\psi}\left(r \frac{v}{|v|}\right)\right]+\sigma \Delta_{v}\left[\widetilde{\psi}\left(r \frac{v}{|v|}\right)\right] \\
& +(v-u[F]) \cdot \frac{\int_{v^{\prime} \neq 0} \nabla_{v^{\prime}}\left[\widetilde{\psi}\left(r \frac{v^{\prime}}{\left|v^{\prime}\right|}\right)\right] F \mathrm{~d} v^{\prime}}{\int_{\mathbb{R}^{d}} F \mathrm{~d} v^{\prime}} \\
= & u[F] \cdot \nabla_{v}\left[\widetilde{\psi}\left(r \frac{v}{|v|}\right)\right]+\sigma \Delta_{v}\left[\widetilde{\psi}\left(r \frac{v}{|v|}\right)\right] \\
& +(v-u[F]) \cdot \int_{r \mathbb{S}^{d-1}}\left(\nabla_{\omega^{\prime}} \widetilde{\psi}\right)\left(\omega^{\prime}\right) M_{l \Omega}\left(\omega^{\prime}\right) \mathrm{d} \omega^{\prime}, \quad v \neq 0 .
\end{aligned}
$$


As $\widetilde{\psi}$ is a collision invariant, we have $\chi(\omega)=0$, for any $\omega \in r \mathbb{S}^{d-1}$, cf. (2.15). Thanks to Lemma [2.5, the integral $I[\widetilde{\psi}]$ can be written

$$
\begin{aligned}
I[\widetilde{\psi}] & =\int_{v \neq 0} \chi(v) F^{(1)} \mathrm{d} v=\left.\frac{\sigma}{\beta} \frac{M_{l \Omega}}{M} \frac{\mathrm{d}}{\mathrm{d} t}\right|_{t=r} \int_{\left|\omega_{t}\right|=t} M\left(\omega_{t}\right) \frac{\chi\left(\omega_{t}\right)}{t\left(t^{2}-r^{2}\right)} \mathrm{d} \omega_{t} \\
& =\left.\frac{\sigma}{\beta} \frac{M_{l \Omega}}{M} \frac{\mathrm{d}}{\mathrm{d} t}\right|_{t=r} \int_{|\omega|=r} M\left(t \frac{\omega}{r}\right) \frac{\chi\left(t \frac{\omega}{r}\right)}{t\left(t^{2}-r^{2}\right)}\left(\frac{t}{r}\right)^{d-1} \mathrm{~d} \omega .
\end{aligned}
$$

Thanks to the second statement in Lemma 2.2, we can write

$$
\nabla_{v}\left[\widetilde{\psi}\left(r \frac{v}{|v|}\right)\right]\left(t \frac{\omega}{r}\right)=\frac{r}{t}\left(\nabla_{\omega} \widetilde{\psi}\right)(\omega)
$$

and by 2.4 in Lemma 2.2(4), we have

$$
\Delta_{v}\left[\widetilde{\psi}\left(r \frac{v}{|v|}\right)\right]\left(t \frac{\omega}{r}\right)=\left(\frac{r}{t}\right)^{2}\left(\Delta_{\omega} \widetilde{\psi}\right)(\omega) .
$$

Therefore, the function $t \rightarrow \chi\left(t \frac{\omega}{r}\right)$ is given by

$$
\chi\left(t \frac{\omega}{r}\right)=\frac{r}{t} u[F] \cdot\left(\nabla_{\omega} \widetilde{\psi}\right)(\omega)+\sigma \frac{r^{2}}{t^{2}}\left(\Delta_{\omega} \widetilde{\psi}\right)(\omega)+\left(t \frac{\omega}{r}-u[F]\right) \cdot W[\widetilde{\psi}],
$$

with $W[\widetilde{\psi}]=\int_{r \mathbb{S}^{d-1}} \nabla_{\omega} \widetilde{\psi} M_{l \Omega}(\omega) \mathrm{d} \omega$. As $\chi(\omega)=0, \omega \in r \mathbb{S}^{d-1}$, because $\widetilde{\psi}$ is a collision invariant, we obtain

$$
\begin{aligned}
M\left(t \frac{\omega}{r}\right) \frac{\chi\left(t \frac{\omega}{r}\right)}{t\left(t^{2}-r^{2}\right)} \\
=M\left(t \frac{\omega}{r}\right) \frac{\chi\left(t \frac{\omega}{r}\right)-\chi(\omega)}{t\left(t^{2}-r^{2}\right)} \\
=M\left(t \frac{\omega}{r}\right) \frac{\frac{r-t}{t} u[F] \cdot\left(\nabla_{\omega} \widetilde{\psi}\right)(\omega)+\sigma \frac{r^{2}-t^{2}}{t^{2}}\left(\Delta_{\omega} \widetilde{\psi}\right)(\omega)+\frac{t-r}{r} \omega \cdot W[\widetilde{\psi}]}{t(t-r)(t+r)} \\
=M\left(t \frac{\omega}{r}\right) \frac{\omega \cdot W[\widetilde{\psi}]}{r t(t+r)}-M\left(t \frac{\omega}{r}\right) \frac{\sigma}{t^{3}}\left(\Delta_{\omega} \widetilde{\psi}\right)(\omega)-M\left(t \frac{\omega}{r}\right) \frac{u[F] \cdot\left(\nabla_{\omega} \widetilde{\psi}\right)(\omega)}{t^{2}(t+r)} \\
=\frac{M\left(t \frac{\omega}{r}\right)}{r t(t+r)}\left[\omega \cdot W[\widetilde{\psi}]+u[F] \cdot\left(\nabla_{\omega} \widetilde{\psi}\right)(\omega)\right]-\frac{\sigma}{t^{3}} \operatorname{div}_{\omega}\left(M\left(t \frac{\omega}{r}\right) \nabla_{\omega} \widetilde{\psi}\right) .
\end{aligned}
$$

It is easily seen that $\int_{r \mathbb{S}^{d-1}} M\left(t \frac{\omega}{r}\right) \omega \mathrm{d} \omega \in \mathbb{R} \Omega$ and, as we know that $W[\widetilde{\psi}] \in(\mathbb{R} \Omega)^{\perp}$, we deduce that

$$
\int_{r \mathbb{S}^{d-1}} M\left(t \frac{\omega}{r}\right) \omega \cdot W[\widetilde{\psi}] \mathrm{d} \omega=0 .
$$

Taking into account that

$$
\int_{r \mathbb{S}^{d-1}} \operatorname{div}_{\omega}\left\{M\left(t \frac{\omega}{r}\right) \nabla_{\omega} \widetilde{\psi}\right\} \mathrm{d} \omega=0
$$


we deduce that

$$
\begin{aligned}
I[\widetilde{\psi}]= & \left.\frac{\sigma}{\beta} \frac{M_{l \Omega}}{M} \frac{\mathrm{d}}{\mathrm{d} t}\right|_{t=r}\left[\left(\frac{t}{r}\right)^{d-1} \int_{r \mathbb{S}^{d-1}} \frac{M\left(t \frac{\omega}{r}\right) \nabla_{\omega} \widetilde{\psi} \cdot u[F]}{r t(t+r)} \mathrm{d} \omega\right] \\
= & \left.\frac{\sigma}{\beta} \frac{M_{l \Omega}}{M} \frac{\mathrm{d}}{\mathrm{d} t}\right|_{t=r}\left[\left(\frac{t}{r}\right)^{d-1} \frac{1}{r t(t+r)}\right] \int_{r \mathbb{S}^{d-1}} M(\omega) \nabla_{\omega} \widetilde{\psi} \cdot u[F] \mathrm{d} \omega \\
& +\left.\frac{\sigma}{2 r^{3} \beta} \frac{M_{l \Omega}}{M} \frac{\mathrm{d}}{\mathrm{d} t}\right|_{t=r} \int_{r \mathbb{S}^{d-1}} M\left(t \frac{\omega}{r}\right) \nabla_{\omega} \widetilde{\psi} \cdot u[F] \mathrm{d} \omega .
\end{aligned}
$$

As before

$$
\frac{M_{l \Omega}}{M} \int_{r \mathbb{S}^{d-1}} M(\omega) \nabla_{\omega} \widetilde{\psi} \mathrm{d} \omega \cdot u[F]=\int_{r \mathbb{S}^{d-1}} M_{l \Omega} \nabla_{\omega} \widetilde{\psi} \mathrm{d} \omega \cdot u[F]=W[\widetilde{\psi}] \cdot u[F]=0
$$

implying that

$$
\begin{aligned}
I[\widetilde{\psi}] & =\left.\frac{\sigma}{2 r^{3} \beta} \frac{M_{l \Omega}}{M} \frac{\mathrm{d}}{\mathrm{d} t}\right|_{t=r} \int_{r \mathbb{S}^{d-1}} M\left(t \frac{\omega}{r}\right) \nabla_{\omega} \widetilde{\psi} \cdot u[F] \mathrm{d} \omega \\
& =\frac{\sigma}{2 r^{3} \beta} \frac{M_{l \Omega}}{M} \int_{r \mathbb{S}^{d-1}} M(\omega)\left(\frac{u[F]-\omega}{\sigma} \cdot \frac{\omega}{r}\right)\left(\nabla_{\omega} \widetilde{\psi} \cdot u[F]\right) \mathrm{d} \omega \\
& =\frac{1}{2 r^{4} \beta} \int_{r \mathbb{S}^{d-1}} M_{l \Omega}\left(u[F] \cdot \omega-r^{2}\right)\left(\nabla_{\omega} \widetilde{\psi} \cdot u[F]\right) \mathrm{d} \omega \\
& =\frac{1}{2 r^{4} \beta} \int_{r \mathbb{S}^{d-1}} M_{l \Omega}\left(\nabla_{\omega} \widetilde{\psi} \cdot u[F]\right)(\omega \cdot u[F]) \mathrm{d} \omega .
\end{aligned}
$$

In the last equality we have used one more time that $W[\widetilde{\psi}] \cdot u[F]=0$. We claim that the last integral vanishes. Indeed, multiplying by $(\omega \cdot u[F])^{2}$ Eq. (3.1) satisfied by the collision invariant $\widetilde{\psi}$ one gets

$$
\begin{aligned}
& 2 \sigma \int_{r \mathbb{S}^{d-1}} M_{l \Omega}\left(\nabla_{\omega} \widetilde{\psi} \cdot u[F]\right)(\omega \cdot u[F]) \mathrm{d} \omega \\
& \quad=W[\widetilde{\psi}] \cdot \int_{r \mathbb{S}^{d-1}} M_{l \Omega}(\omega \cdot u[F])^{2}(\omega-u[F]) \mathrm{d} \omega \\
& \quad=W[\widetilde{\psi}] \cdot \int_{r \mathbb{S}^{d-1}} M_{l \Omega}(\omega \cdot u[F])^{2} \omega \mathrm{d} \omega .
\end{aligned}
$$

It is easily seen that $\int_{r \mathbb{S}^{d-1}} M_{l \Omega}(\omega \cdot u[F])^{2} \omega \mathrm{d} \omega \in \mathbb{R} \Omega$ and therefore

$$
W[\widetilde{\psi}] \cdot \int_{r \mathbb{S}^{d-1}} M_{l \Omega}(\omega \cdot u[F])^{2} \omega \mathrm{d} \omega=0,
$$

saying that $I[\widetilde{\psi}]=0$. 


\section{Acknowledgments}

J.A.C. acknowledges partial support of the Royal Society via a Wolfson Research Merit Award and the EPSRC grant EP/P031587/1. M.B. acknowledges support from the Euratom Research and Training programme 2014-2018 under grant agreement No. 633053.

\section{References}

1. M. Abramowitz and I. Stegun, Handbook of Mathematical Functions: With Formulas, Graphs and Mathematical Tables (Dover Books on Mathematics, 1965).

2. M. Agueh, R. Illner and A. Richardson, Analysis and simulations of a refined flocking and swarming model of Cucker-Smale type, Kinet. Relat. Models 4 (2011) 1-16.

3. G. Albi, D. Balagué, J. A. Carrillo and J. von Brecht, Stability analysis of flock and mill rings for second order models in swarming, SIAM J. Appl. Math. 74 (2014) 794-818.

4. I. Aoki, A simulation study on the schooling mechanism in fish, Bull. Japan Soc. Sci. Fisheries 48 (1982) 1081-1088.

5. A. B. T. Barbaro, J. A. Cañizo, J. A. Carrillo and P. Degond, Phase transitions in a kinetic flocking model of Cucker-Smale type, Multiscale Model. Simulat. 14 (2016) 1063-1088.

6. A. B. T. Barbaro, B. Einarsson, B. Birnir, S. Sigurthsson, H. Valdimarsson, O. K. Palsson, S. Sveinbjornsson and T. Sigurthsson, Modelling and simulations of the migration of pelagic fish, ICES J. Mar. Sci. 66 (2009) 826-838.

7. A. B. T. Barbaro, K. Taylor, P. F. Trethewey, L. Youseff and B. Birnir, Discrete and continuous models of the dynamics of pelagic fish: Application to the capelin, Math. Comput. Simulat. 79 (2009) 3397-3414.

8. A. L. Bertozzi, T. Kolokolnikov, H. Sun, D. Uminsky and J. von Brecht, Ring patterns and their bifurcations in a nonlocal model of biological swarms, Commun. Math. Sci. 13 (2015) 955-985.

9. F. Bolley, J. A. Cañizo and J. A. Carrillo, Stochastic mean-field limit: Non-Lipschitz forces and swarming, Math. Models Methods Appl. Sci. 21 (2011) 2179-2210.

10. F. Bolley, J. A. Cañizo and J. A. Carrillo, Mean-field limit for the stochastic Vicsek model, Appl. Math. Lett. 25 (2012) 339-343.

11. M. Bostan, The Vlasov-Poisson system with strong external magnetic field. Finite Larmor radius regime, Asymptot. Anal. 61 (2009) 91-123.

12. M. Bostan, Transport equations with disparate advection fields. Application to the gyrokinetic models in plasma physics, J. Differential Equations 249 (2010) 16201663.

13. M. Bostan, Gyro-kinetic Vlasov equation in three dimensional setting. Second order approximation, SIAM J. Multiscale Model. Simulat. 8 (2010) 1923-1957.

14. M. Bostan, Transport of charged particles under fast oscillating magnetic fields, SIAM J. Math. Anal. 44 (2012) 1415-1447.

15. M. Bostan, On the Boltzmann equation for charged particle beams under the effect of strong magnetic fields, Discrete Contin. Dynam. Syst. Ser. B 20 (2015) 339-371.

16. M. Bostan, High magnetic field equilibria for the Fokker-Planck-Landau equation, Ann. Inst. H. Poincaré Anal. Non Linéaire 33 (2016) 899-931, http://dx.doi.org/ 10.1016/j.anihpc.2015.01.008.

17. M. Bostan, Strongly anisotropic diffusion problems: Asymptotic analysis, J. Differential Equations 256 (2016) 1043-1092. 
18. M. Bostan and C. Caldini-Queiros, Finite Larmor radius approximation for collisional magnetic confinement. Part I: The linear Boltzmann equation, Quart. Appl. Math. 72 (2014) 323-345.

19. M. Bostan and C. Caldini-Queiros, Finite Larmor radius approximation for collisional magnetic confinement. Part II: The Fokker-Planck-Landau equation, Quart. Appl. Math. 72 (2014) 513-548.

20. M. Bostan and J.-A. Carrillo, Asymptotic fixed-speed reduced dynamics for kinetic equations in swarming, Math. Models Methods Appl. Sci. 23 (2013) 2353-2393.

21. W. Braun and K. Hepp, The Vlasov dynamics and its fluctuations in the $1 / N$ limit of interacting classical particles, Commun. Math. Phys. 56 (1977) 101-113.

22. H. Brézis, Analyse Fonctionnelle: Théorie et Applications (Masson, 1983).

23. J. A. Cañizo, J. A. Carrillo and J. Rosado, A well-posedness theory in measures for some kinetic models of collective motion, Math. Models Methods Appl. Sci. 21 (2011) 515-539.

24. J. A. Carrillo, Y.-P. Choi and M. Hauray, The derivation of swarming models: Meanfield limit and Wasserstein distances, in Collective Dynamics from Bacteria to Crowds: An Excursion through Modeling, Analysis and Simulation, CISM International Centre for Mechanical Sciences, Vol. 533 (Springer, 2014), pp. 1-45.

25. J. A. Carrillo, Y.-P. Choi and M. Hauray, Local well-posedness of the generalized Cucker-Smale model with singular kernels, ESAIM Proc. 47 (2014) 17-35.

26. J. A. Carrillo, Y.-P. Choi, M. Hauray and S. Salem, Mean-field limit for collective behavior models with sharp sensitivity regions, to appear in J. European Math. Soc.

27. J. A. Carrillo, M. R. D'Orsogna and V. Panferov, Double milling in a self-propelled swarms from kinetic theory, Kinet. Relat. Models 2 (2009) 363-378.

28. J. A. Carrillo, M. Fornasier, J. Rosado and G. Toscani, Asymptotic flocking dynamics for the kinetic Cucker-Smale model, SIAM J. Math. Anal. 42 (2010) 218-236.

29. J. A. Carrillo, M. Fornasier, G. Toscani and F. Vecil, Particle, kinetic, and hydrodynamic models of swarming, in Mathematical Modeling of Collective Behavior in Socio-Economic and Life Sciences, Modelling and Simulation in Science and Technology (Birkhäuser, 2010), pp. 297-336.

30. J. A. Carrillo, Y. Huang and S. Martin, Explicit flock solutions for quasi-Morse potentials, European J. Appl. Math. 25 (2014) 553-578.

31. J. A. Carrillo, Y. Huang and S. Martin, Nonlinear stability of flock solutions in secondorder swarming models, Nonlinear Anal. Real World Appl. 17 (2014) 332-343.

32. J. A. Carrillo, A. Klar, S. Martin and S. Tiwari, Self-propelled interacting particle systems with roosting force, Math. Models Methods Appl. Sci. 20 (2010) 1533-1552.

33. J. A. Carrillo, A. Klar and A. Roth, Single to double mill small noise transition via semi-Lagrangian finite volume methods, Commun. Math. Sci. 14 (2016) 1111-1136.

34. Y.-L. Chuang, M. R. D'Orsogna, D. Marthaler, A. L. Bertozzi and L. Chayes, State transitions and the continuum limit for a $2 \mathrm{D}$ interacting, self-propelled particle system, Physica D 232 (2007) 33-47.

35. Y.-L. Chuang, Y. R. Huang, M. R. D’Orsogna and A. L. Bertozzi, Multi-vehicle flocking: Scalability of cooperative control algorithms using pairwise potentials, in IEEE Int. Conf. Robotics and Automation (IEEE, 2007), pp. 2292-2299.

36. I. D. Couzin, J. Krause, N. R. Franks and S. A. Levin, Effective leadership and decision making in animal groups on the move, Nature 433 (2005) 513-516.

37. I. D. Couzin, J. Krause, R. James, G. D. Ruxton and N. R. Franks, Collective memory and spatial sorting in animal groups, J. Theor. Biol. 218 (2002) 1-11.

38. F. Cucker and S. Smale, On the mathematics of emergence, Japan J. Math. 2 (2007) $197-227$. 
39. F. Cucker and S. Smale, Emergent behavior in flocks, IEEE Trans. Automat. Control 52 (2007) 852-862.

40. A. Czirok, H. E. Stanley and T. Vicsek, Spontaneously ordered motion of selfpropelled particles, J. Phys. A: Math. Gen. 30 (1997) 1375-1385.

41. P. Degond, A. Frouvelle and J.-G. Liu, Macroscopic limits and phase transition in a system of self-propelled particles, J. Nonlinear Sci. 23 (2013) 427-456.

42. P. Degond, A. Frouvelle and J.-G. Liu, Phase transitions, hysteresis, and hyperbolicity for self-organized alignment dynamics, Arch. Ration. Mech. Anal. 216 (2015) 63-115.

43. P. Degond, A. Frouvelle and S. Merino-Aceituno, A new flocking model through body attitude coordination, arXiv: 1605.03509.

44. P. Degond, A. Frouvelle, S. Merino-Aceituno and A. Trescases, Quaternions in collective dynamics, arXiv: 1701.01166.

45. P. Degond, J.-G. Liu, S. Motsch, V. Panferov, Hydrodynamic models of self-organized dynamics: Derivation and existence theory, Methods Appl. Anal. 20 (2013) 89-114.

46. P. Degond, J.-G. Liu and C. Ringhofer, Evolution of wealth in a nonconservative economy driven by local Nash equilibria, Philos. Trans. Roy. Soc. A 372 (2014) 20130394.

47. P. Degond, A. Manhart and $\mathrm{H}$. Yu, A continuum model for nematic alignment of self-propelled particles, arXiv: 1509.03124.

48. P. Degond and S. Motsch, Continuum limit of self-driven particles with orientation interaction, Math. Models Methods Appl. Sci. 18 (2008) 1193-1215.

49. R. Dobrushin, Vlasov equations, Funct. Anal. Appl. 13 (1979) 115-123.

50. M. R. D'Orsogna, Y.-L. Chuang, A. L. Bertozzi and L. Chayes, Self-propelled particles with soft-core interactions: Patterns, stability and collapse, Phys. Rev. Lett. 96 (2006) 104302-1/4.

51. W. Ebeling and U. Erdmann, Nonequilibrium statistical mechanics of swarms of driven particles, Complexity 8 (2003) 23-30.

52. A. Frouvelle and J.-G. Liu, Dynamics in a kinetic model of oriented particles with phase transition, SIAM J. Math. Anal. 44 (2012) 791-826.

53. G. Grégoire and H. Chaté, On set of collective and cohesive motion, Phys. Rev. Lett. 92 (2004) 025702-1/4.

54. S.-Y. Ha, K. Lee and D. Levy, Emergence of time-asymptotic flocking in a stochastic Cucker-Smale system, Commun. Math. Sci. 7 (2009) 453-469.

55. S.-Y. Ha and J.-G. Liu, A simple proof of the Cucker-Smale flocking dynamics and mean-field limit, Commun. Math. Sci. 7 (2009) 297-325.

56. S.-Y. Ha and E. Tadmor, From particle to kinetic and hydrodynamic descriptions of flocking, Kinet. Relat. Models 1 (2008) 415-435.

57. H. Hildenbrandt, C. Carere and C. K. Hemelrijk, Self-organised complex aerial displays of thousands of starlings: A model, Behav. Ecol. 21 (2010) 1349-1359.

58. A. Huth and C. Wissel, The simulation of the movement of fish schools, J. Theor. Biol. 156 (1992) 365-385.

59. T. Kolokolnikov, J. A. Carrillo, A. Bertozzi, R. Fetecau and M. Lewis, Emergent behaviour in multi-particle systems with non-local interactions, Physica D 260 (2015) $1-4$.

60. H. Levine, W.-J. Rappel and I. Cohen, Self-organization in systems of self-propelled particles, Phys. Rev. E 63 (2000) 017101-1/4.

61. S. Motsch and E. Tadmor, A new model for self-organized dynamics and its flocking behavior, J. Statist. Phys. 144 (2011) 923-947.

62. S. Motsch and E. Tadmor, Heterophilious dynamics enhances consensus, SIAM Rev. 56 (2014) 577-621. 
63. H. Neunzert, The Vlasov equation as a limit of Hamiltonian classical mechanical systems of interacting particles, Trans. Fluid Dynam. 18 (1977) 663-678.

64. J. Parrish and L. Edelstein-Keshet, Complexity, pattern and evolutionary trade-offs in animal aggregation, Science 294 (1999) 99-101.

65. C. W. Reynolds, Flocks, herds and schools: A distributed behavioral model, ACM SIGGRAPH Comput. Graph. 21 (1987) 25-34.

66. T. Vicsek, A. Czirok, E. Ben-Jacob, I. Cohen and O. Shochet, Novel type of phase transition in a system of self-driven particles, Phys. Rev. Lett. 75 (1995) 1226-1229. 